\title{
Job Insecurity
}

\section{By Aditya Kuvalekar And Elliot LiPNOwsk]*}

We examine the relationship between job security and productivity in a fixed wage worker-firm relationship facing match quality uncertainty. The worker's action affects both learning and current productivity. The firm, seeing worker behavior and outcomes, makes a firing decision. As bad news accrues, the firm cannot commit to retain the worker. This creates perverse incentives: the worker strategically slows learning, harming productivity. We fully characterize the unique equilibrium in our continuous-time game. Consistent with some evidence in organizational psychology, the relationship between job insecurity and productivity is $U$-shaped: a worker is least productive when his job is moderately secure. (JEL J23, J24, J63, M51)

wide body of literature, from economics and management to organizational psychology, studies the effects of job insecurity on employee productivity. The results are ambiguous. Repenning (2000) alludes to two competing schools of thought regarding the consequences of job insecurity. The "drive in fear" school argues that fear of job loss can boost productivity by motivating employees, while the "drive out fear" school argues that such fear distorts decision-making and thus harms productivity.

In Only the Paranoid Survive, Intel CEO Andy Grove extols the virtues of fear in organizational decision-making:

The most important role of managers is to create an environment in which people are passionately dedicated to winning in the marketplace. Fear plays a major role in creating and maintaining such passion ... Simply put, fear can be the opposite of complacency.

\footnotetext{
* Kuvalekar: Universidad Carlos III de Madrid, C/Madrid 126, Getafe 28903 Spain (email: akuvalek@eco. uc3m.es); Lipnowski: Department of Economics, Columbia University, 420 West 118th Street, New York, NY 10027 (email: e.lipnowski@ columbia.edu). Michael Ostrovsky was coeditor for this article. We are especially grateful to Joyee Deb, David Pearce, Debraj Ray, Maher Said, and Ennio Stacchetti for advice and feedback. We also thank Dan Bernhardt, Alessandro Bonatti, René Caldentey, Boyan Jovanovic, Robert Kohn, Antoine Loeper, Derek Neal, Mikhail Panov, João Ramos, Philipp Strack, Srinivasa Varadhan, Dong Wei, and an anonymous referee, as well as audiences at Midwest Theory, Stony Brook, and SITE. Kuvalekar gratefully acknowledges support from the Ministerio Economia y competitividad grant PGC2018-09159-B-I00 and Comunidad de Madrid, MadEco-CM (S2015/HUM-3444) grant.

Go to https://doi.org/10.1257/mic.20190132 to visit the article page for additional materials and author disclosure statement $(\mathrm{s})$ or to comment in the online discussion forum.
} 
On the other hand, among W. Edwards Deming's $(1982,59) 14$ key management principles is

Drive out fear. No one can put in his best performance unless he feels secure ... A common denominator of fear in any form, anywhere, is loss from impaired performance and padded figures.

Fear has real negative consequences, Deming argues, like the employee who "[would] like to understand better the reasons for some of the company's procedures, but [doesn't] dare to ask about them (Deming 1982, 61)." In short, fear induces employees to be overly cautious, impeding their productivity.

Empirical work, by both economists and organizational psychologists, has also found an ambiguous relationship between productivity and job insecurity. Some organizational psychologists find positive associations (e.g., Probst et al. 2007; Brockner, Tyler, and Cooper-Schneider 1992; Sverke and Hellgren 2001), some negative (e.g., Gilboa et al. 2008, Roskies and Louis-Guerin 1990, Reisel et al. 2010), and some, a more complex relationship (e.g., Staufenbiel and König 2010). One study (Selenko et al. 2013) seems to find a U-shaped relationship: a worker is least productive when his job is moderately secure.

Motivated by the above, our goal is to explore the relationship between job insecurity and productivity in an agency framework. To this end, we provide a simple framework of a fixed-wage firm-worker relationship, where the worker faces a firing threat. ${ }^{1}$ A key feature of our model is that employees are sometimes fired for poor performance, as past performance heralds future performance. In our model, job insecurity and productivity are endogenously codetermined: fear of job loss feeds into worker decisions, and productivity feeds into firing choices. We show that this interplay yields a U-shaped relationship between employee productivity and job insecurity.

Model.-We study an infinite horizon, continuous time game between a worker and a firm. At any given moment, the firm decides whether or not to continue employing the worker at a fixed flow cost. While employed, the worker collects a fixed wage, and chooses an action. The flow of output is stochastically determined by the worker's action and a relationship-specific "match quality" between the worker and the firm. The players are symmetrically uninformed about the match quality: they both learn from observing the output and the action. Higher actions generate both higher expected output and superior information about match quality, and so the firm prefers that the worker chooses higher actions..$^{2}$ We study Markov perfect equilibrium of this game, with players' beliefs about match quality as the state.

The players have conflicting interests. The worker is indifferent to firm output and his own action, wanting only to stay employed as long as possible. The

\footnotetext{
${ }^{1}$ According to the US Bureau of Labor Statistics (see http://www.bls.gov/opub/reports/minimum-wage/ archive/characteristics-of-minimum-wage-workers-2014.pdf), 58.7 percent of wage and salary workers are paid a fixed hourly wage. Thus, we focus on environments without incentive pay, just as Shapiro and Stiglitz (1984) did.

${ }^{2}$ While maintained throughout the paper for ease of exposition, this assumption is not strictly necessary. See Section IVA for a more general model that generates our key results.
} 
firm, however, maximizes profit, and so weighs its expectations of future output against the cost of employing the worker. The firm finds it profitable to employ a worker if and only if the two are well-matched.

An important feature of our model is that the worker's choices are perfectly observable, and yet the firm cannot write contingent contracts. ${ }^{3}$ This yields a more tractable model: no player ever has private information (on or off path), so that their beliefs about match quality always agree. Given this feature, one can view our model as a collective experimentation problem with conflicting interests between the firm and the worker. In that sense, our model is substantially related to that of Garfagnini (2011), who considers an experimentation problem that is jointly carried out by two players. Several substantive modeling differences yield very different dynamics, and in particular rule out the variation in productivity over time. ${ }^{4}$

Results.-We show that our players' relationship entails a severe agency problem and exhibits rich dynamics. Even with the worker's actions being observable to the firm and him being indifferent across actions, a nontrivial agency problem arises. Our first main result, Proposition 1, shows that in equilibrium, the firm's value is as if the worker chose the lowest action at all histories. Thus, the enhanced efficiency from firing unproductive workers comes at a cost, as formalized by the proposition: by distorting incentives, it results in "worse" worker behavior. ${ }^{5}$ This inefficiency is the outcome of two distinguishing features of our model: the firm cannot commit to retain an unsuccessful worker, and the worker faces no high-powered reward for success. Collectively, these imply that the worker does not want to create information for the firm. Proposition 1 tells us that the agency problem is severe; however, it does not tell us how our players actually behave.

Our next main result, Theorem 1, fully describes equilibrium, and shows it is unique. Equilibrium play is characterized by three regimes. When the players are pessimistic about match quality, the firm immediately fires the worker. When the players are optimistic about match quality, the firm continues to employ the worker. At such beliefs, enjoying a sense of job security, the worker seeks to "avoid making waves" for fear of risking his high status in the firm: he chooses the lowest available action. For moderate beliefs, the firm sporadically fires the worker, who in turn chooses a higher action than when his job is secure.

As can be seen in Figure 1, the equilibrium exhibits discontinuous behavior by both players. The intuition behind this feature comes directly from the firm's incentives at the cutoff where the probationary and optimistic regions meet. In light of Proposition 1, this cutoff is the firm's optimal stopping belief against a worker

\footnotetext{
${ }^{3}$ Of course, if our firm could write such contracts, the situation would be fundamentally nonstrategic; given observable action, the firm could dictate the worker's behavior under the threat of firing.

${ }^{4}$ In the "observable effort" model of Garfagnini (2011) there is an agent who exerts costly effort and cares about the state of the world. His experimentation technology is of the perfectly revealing "good news" Poisson form rather than Brownian. His principal can condition his stopping choice on the agent's current effort rather than only on beliefs. If his agent exerts zero effort, experimentation halts completely and, crucially, his principal does strictly worse than under no experimentation. Given the last feature, no analogue of Corollary 1 can obtain in the world of Garfagnini (2011); see footnote 22.

${ }^{5}$ Lazear (1990) argues that reducing employment protection can lead to lower unemployment by making firms more efficient. In our model, there is also an associated efficiency loss.
} 


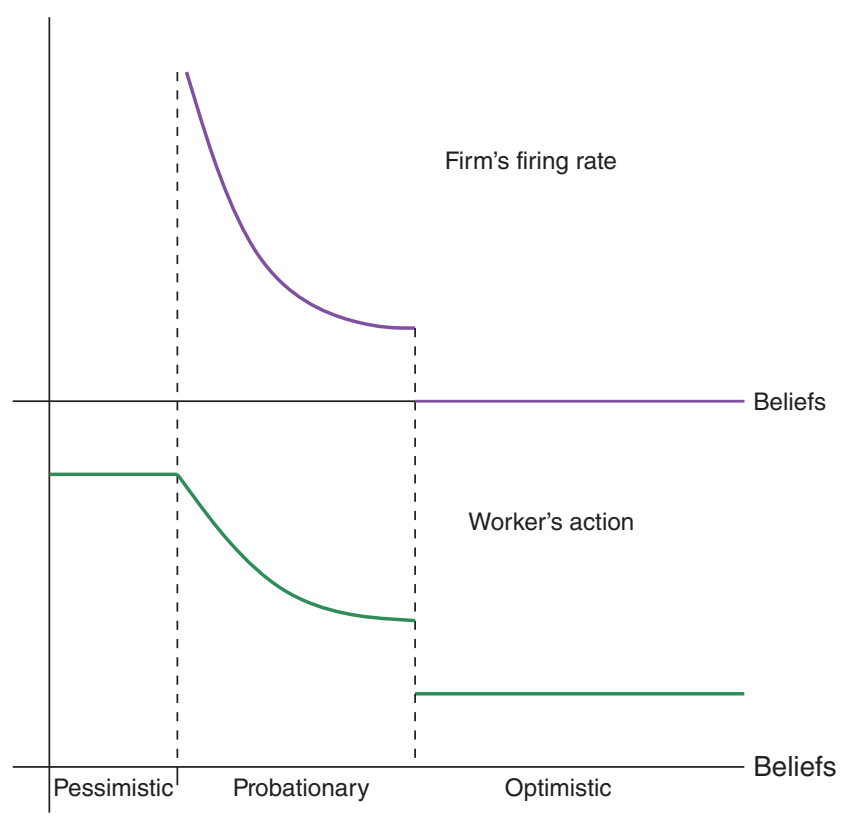

Figure 1. Players' Strategies

strategy of constant (low) action. Standard option value reasoning then tells us that the firm's flow profit is negative just above the cutoff. At the same time, to keep the firm indifferent (and so willing to mix) just below the cutoff, the worker must offer the firm a flow profit of zero, necessitating a strictly higher action. Discontinuity follows.

Our equilibrium features a nonmonotonic relationship between job security (i.e., expected discounted future employment) and productivity (i.e., expected current flow profit). We formalize this in Corollary 1 . Consider how productivity varies as beliefs about match quality—and, with them, the worker's job security-increase. First, in the middle region (which one can interpret as a probationary region), the worker barely earns his keep, so that productivity is constant. ${ }^{6}$ As beliefs pass to the (barely) optimistic region, the worker discontinuously switches to the minimum action: he is now less productive. As beliefs grow further through the optimistic region, the worker's action remains constant, but his expected match quality with the firm increases: he is in expectation more productive. So productivity first decreases with job security due to changing worker incentives and then increases with it due to worker selection. Thus, our equilibrium provides plausible mechanics that generate a U-shaped relationship.

Moreover, our results may speak to the management debate we reference above. Our middle region, with intermittent firing and higher actions than that suggested by Proposition 1, tells us why one might "drive in fear": the threat of firing the

\footnotetext{
${ }^{6}$ Note, in the pessimistic region, there is no job to lose.
} 


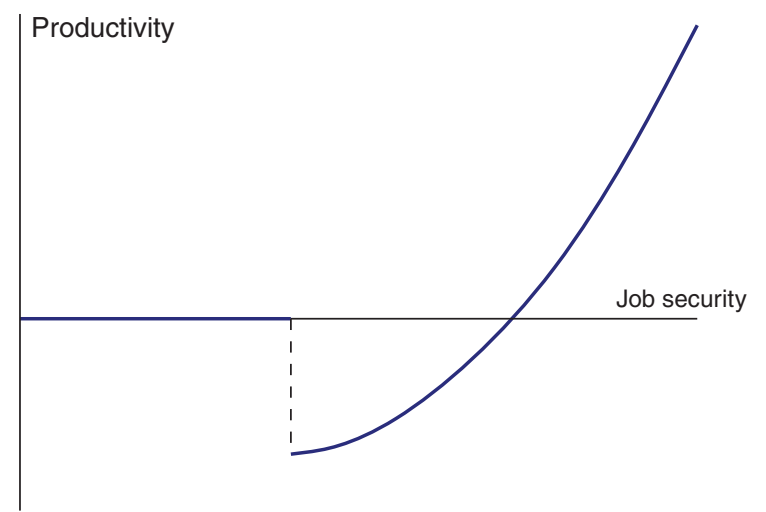

Figure 2. Observed Relationship between Job Security and Productivity

worker faces at his current status motivates him to work harder. Our optimistic region highlights the reason to "drive out fear." In this region, the worker chooses the low action, but not because his job is safe. Rather, he deliberately slows the flow of information for fear of entering the more pessimistic regime: future job insecurity creates perverse current incentives for the worker.7]

In addition to our equilibrium characterization and its consequences for the firm, our analysis may be of broader interest for the study of dynamic games. In single agent settings, the vast literature on optimal control and optimal stopping yields powerful methods for understanding well-behaved dynamic optimization problems in continuous time. ${ }^{8}$ Standard results from this literature, however, rely on the decision-maker facing an environment that varies smoothly with the underlying state. In strategic settings, one player's best response may change discontinuously with the state, and so we may be forced to study the resulting discontinuous environment faced by the other player. Some, but not all, past work avoids this issue in modeling strategic settings with only one "large" strategic player. ${ }^{9}$ In our setting, we extend known smooth pasting techniques to characterize equilibrium when multiple large players' equilibrium actions may vary discontinuously. In a similar vein, we provide a new refinement, termed instantaneous sequential rationality, for continuous time games. ${ }^{10}$ By characterizing its behavioral implications in our game, we show that it can be a useful refinement, reducing a continuum family of qualitatively similar equilibria to a unique equilibrium (which is Pareto optimal within the family).

\footnotetext{
${ }^{7}$ Indeed, if the firm's strategy were to employ the worker forever-which it could not do in equilibrium-the worker would willingly choose the high action in our setting.

${ }^{8}$ See Pham (2009), for example.

${ }^{9}$ This assumption is a natural one when one agent has all of the market power. For examples of such settings, see Daley and Green (2012) and Board and Meyer-ter-Vehn (2013). Other settings, such as those of Bolton and Harris (1999) and Daley and Green (2020), have multiple large players and yet exhibit continuous equilibrium play, ruling out this potential difficulty.

${ }^{10}$ See Section III for more details.
} 
Robustness of Results.-To isolate the relationship between job security and productivity for a single worker-firm relationship, we have assumed that actions are costless, that the worker's choice is one-dimensional, that the worker cannot be promoted to provide incentives, and that the worker's wage level does not respond to his current marginal product. The extensions outlined in Section IV show that our qualitative results are robust to all of these features. With higher actions being costly — and thus interpretable as effort-behavior off of the path of play may differ from ours, but every equilibrium generates dynamics qualitatively similar to ours; similar equilibrium dynamics also obtain if the worker's action is multidimensional. If the worker is paid his current marginal product as in Holmström (1999)—or any other wage exhibiting sufficiently low-powered incentives-equilibrium play is identical. If, in a common nonpecuniary instance of low-powered incentives, the worker is promoted when past success is above a threshold, then equilibrium play is richer: the worker chooses the high action when near enough to promotion, but the non-monotonicity of Corollary 1 persists.

The remainder of the paper proceeds as follows. In Section I, we describe our game and define our equilibrium concept. Section II presents and discusses our main results, fully characterizing our unique equilibrium. In Section III, we describe our broader contribution to the study of dynamic games in continuous time. Section IV details extensions of our model. All proofs are in the Appendix.

\section{Our Model}

\section{A. The Environment}

We study an infinite horizon, continuous time game between a firm and a worker. At each instant, the firm decides whether or not to irreversibly fire the worker, while the worker chooses an action conditional on not being fired.

The worker-firm match quality $\theta \in\{0,1\}$ is symmetrically unknown to the players, with prior $E_{0} \theta=p_{0} \in(0,1)$. Irrespective of the worker's action, the stochastic output is profitable on average if and only if he is well-matched with the firm (something neither the firm nor the worker knows). The worker's choice of action, along with his productive outcomes, are public. The worker wants to stay employed and collect his fixed wage, and so cares about the information that actions generate; to isolate the incentive effects of job insecurity, we assume that all actions are costless.

At each instant, the worker makes a choice $A_{t} \in[\underline{a}, \bar{a}]$. Over the course of $[t, t+d t)$, the firm observes this action as well as the resulting (stochastic) output. The players update their beliefs about the match quality, taking into account the action chosen. The firm decides whether to continue the relationship or fire the worker.

We stress that no matter what action the worker chooses, our two players always (on path and off path) have the same information about the match quality, so that there can be no private beliefs. ${ }^{11}$

\footnotetext{
${ }^{11}$ This stands in contrast to papers on dynamic contracting with (initially) symmetric uncertainty and moral hazard, such as Prat and Jovanovic (2014), Demarzo and Sannikov (2017), and Cisternas (2018a). A paper which
} 


\section{B. Payoffs}

We model the firm's flow profit as a diffusion with drift depending on both the match quality and the worker's action. The firm also incurs a flow cost $c$ of hiring the worker. Let $X_{t}$ denote the cumulative output (net of the cost of hiring the worker). The law of motion of $X$ is then

$$
d X_{t}=\left(\theta A_{t}-c\right) d t+\sigma d B_{t}
$$

where $\left\{B_{t}\right\}_{t}$ is a standard Brownian motion. ${ }^{12}$ The firm's accrued payoff over $[t, t+d t)$ is $d X_{t}$ while employing the worker, and the worker's accrued payoff is equal to his collected wages $w d t$ (where $w>0$ is a fixed wage). It may be natural to assume $w=c$, but it is not necessary. After the worker is fired, both players have continuation payoff normalized to zero. Therefore, if the worker is fired at (stochastic) time $\tau$, the firm's and worker's payoffs are

$$
r \int_{0}^{\tau} e^{-r t} d X_{t} \quad \text { and } \quad r \int_{0}^{\tau} e^{-r t} w d t
$$

respectively.

We assume that $\bar{a}>\underline{a}>c>0$. So the firm would (myopically) strictly want to employ a well matched worker and fire a poorly matched worker. By counting time, profit, and worker values in different units, we may without loss of generality normalize $r=\sigma=w=1$.

\section{Learning}

We assume that the players (symmetrically) revise their beliefs about worker-firm match via Bayesian updating. We assume that players cannot signal what they do not know: nobody draws any direct inferences from the firm's stopping choice or the worker's activity choice. However, the worker's choice $A_{t}$ does indirectly affect learning, as it affects the stochastic law of $d X_{t}$, and it affects the inferences (concerning $\theta$ ) the players draw from $d X_{t}$.

Let $P_{t} \in(0,1)$ be the time $t$ public expectation of $\theta$, i.e., $P_{t}$ is the commonly held belief at time $t$ that the worker and firm are well matched. Sometimes, it is more convenient to work with the state variable $Z_{t}:=\log \left(P_{t} /\left(1-P_{t}\right)\right)$. It is, of course, informationally equivalent: any $z$ is associated with a probability $p(z):=1 /\left(1+e^{-z}\right)$. As noted in Daley and Green (2012) and in Bolton and Harris (1999), the process $Z$ is a linear transformation of the output $X$, and is therefore a Brownian diffusion process. Whenever the firm has not yet stopped, the belief variable $Z$ follows the law of motion

$$
d Z_{t}=A_{t}\left[d X_{t}-\left(\frac{1}{2} A_{t}-c\right) d t\right]
$$

shares our observability assumption is Kostadinov and Kuvalekar (forthcoming), studying symmetric learning in a relational contract.

${ }^{12}$ The exact functional form is not crucial for our qualitative results. See Section IVA for details. 
Equivalently, $P$ follows

$$
\begin{gathered}
d P_{t}=A_{t} P_{t}\left(1-P_{t}\right)\left[d X_{t}-\left(P_{t} A_{t}-c\right) d t\right] \\
=A_{t} P_{t}\left(1-P_{t}\right)\left[A_{t}\left(\theta-P_{t}\right) d t+d B_{t}\right] . \\
\text { D. Player Strategies }
\end{gathered}
$$

Informally, our players both observe the history $\left(A_{\tilde{t}}, X_{\tilde{t}}\right)_{\tilde{t}<t}$, and our worker and firm choose action $A_{t}$ and stopping rate $S_{t}$, respectively (where $S_{t}=\infty$ corresponds to firing the worker with certainty). ${ }^{13}$

We restrict our attention to Markovian strategies using beliefs about the match quality as our state variable. Plainly, this simplifying assumption is inadequate for describing many organizational settings. With access to formal contracts, relational incentives, and other means for actors to tie each others' hands, organizations with the same current information concerning match quality could realistically engage in very different relationships going forward. Having said that, we will show that this extreme modeling assumption enables tractable analysis of a unique equilibrium in our game, which still gives rise to rich relational dynamics. Our hope is that, for organizations that work outside the realm of such devices, our results represent something real about the relationship between job security and productivity. In Subsection IVE, we briefly and informally explore how relational incentives can alter the worker-firm relationship.

The first convenience our Markovian restriction affords is that the stochastic process of players' beliefs is uniquely determined by the realized path of output. Indeed it follows from Nakao (1972) that the law of motion of $Z$ as governed by equation (1) has a unique strong solution when players follow Markov strategies as defined below.

DEFINITION 1: A Markov strategy profile is a pair $(a, s)$, where

(i) $a:[0,1] \rightarrow[\underline{a}, \bar{a}]$ is piecewise Lipschitz.

(ii) $s:[0,1] \rightarrow[0, \infty]$ is piecewise continuous; $s$ is piecewise Lipschitz in any interval $\subseteq[0,1]$ over which $s$ is bounded; and $s^{-1}(\infty)$ is closed.

Let the space of above specified strategies for the worker be $\mathcal{A}$ and for the firm be $\mathcal{S}$.

\footnotetext{
${ }^{13}$ Notice that this formulation rules out the possibility that the firm stops with probability exactly $q \in(0,1)$ at a given history. Given that we will focus entirely on Markov strategies, this is in some sense a normalization. Indeed, as the belief process will have volatility locally bounded away from zero, the set of times $t$ at which $P_{t}$ is $p$ has no isolated points: the belief diffusion will repeatedly quickly revisit $p$. Therefore, if the firm fired the worker with probability $q \in(0,1)$ every time $P_{t}=p$, then the probability of having fired the worker as of time $t+\epsilon$ would be 1 for any $t$ such that $P_{t}=p$ and any $\epsilon>0$.

We conjecture that an analogous argument should rule out any cumulative stopping process that fails to be absolutely continuous, but such an argument is beyond the scope of this paper. Thus, conservatively, we can think of the "stopping rate" form of firm behavior as a smoothness assumption.
} 
Given $P_{0} \in[0,1]$ and $(a, s) \in \mathcal{A} \times \mathcal{S}$, define the induced stochastic processes $\left\{P_{t}, A_{t}, S_{t}\right\}_{t}$ by letting $A_{t}=a\left(P_{t}\right)$ and $S_{t}=s\left(P_{t}\right)$, and letting $\left\{P_{t}\right\}_{t}$ follow equation (2).

In an abuse of notation, we identify any two strategies for a player that agree almost everywhere, and (in the case of the firm) are finite-valued on the same set. For the remainder of this paper, every equation concerning players' behavior (as a function of beliefs) is understood to hold only up to this equivalence.

Certain classes of strategies seem natural. For a fixed action $\hat{a} \in[\underline{a}, \bar{a}]$, the worker has a constant control strategy, with action $\hat{a}$ chosen at every belief; we simply call this strategy $\hat{a} \in \mathcal{A}$. For a belief $p \in[0,1]$, the firm has a simple cutoff strategy $s^{p} \in \mathcal{S}$ with $\left.s_{p}\right|_{(p, 1]}=0$ and $\left.s_{p}\right|_{[0, p]}=\infty$. As is standard, if the worker uses a constant control, then the firm optimally uses a simple cutoff.

By allowing $S_{t} \in(0, \infty)$, we have permitted the firm to mix. That is, whenever $S_{t}>0$, the probability of stopping over $[t, t+d t)$ is approximately $S_{t} d t$. As we will see, this mixing is (for many parameter values) a necessary component of equilibrium. In the present definition of a worker strategy, we allow the worker to observably choose interior actions, but we have ruled out the possibility of explicit mixing. The latter is purely for notational convenience.

\section{E. Defining Equilibrium}

As the stochastic differential equation 1 uniquely pins down the law of motion of beliefs given a strategy profile $(a, s) \in \mathcal{A} \times \mathcal{S}$ and initial condition $P_{0}=p \in[0,1]$, there is no need to separately specify history-dependent beliefs in the specification of an equilibrium.

We can define the worker's value as

$$
v(p \mid a, s)=E_{a, s}\left[\int_{0}^{\infty} e^{-t} e^{-\int_{0}^{t} S_{\tau} d \tau} d t \mid P_{0}=p\right],
$$

where the term $e^{-\int_{0}^{t} S_{\tau} d \tau}$ is understood to be zero when $S_{\tau}=\infty$ for some $\tau \in[0, t)$.

Similarly, the firm's value can be defined as

$$
\pi(p \mid a, s)=E_{a, s}\left[\int_{0}^{\infty} e^{-t} e^{-\int_{0}^{t} S_{\tau} d \tau} d X_{t} \mid P_{0}=p\right]
$$

As is typical, we define equilibrium as a strategy profile satisfying mutual best response. Given our focus on strategies that are Markoyian with respect to beliefs, and given that no proper subset of $(0,1)$ is absorbing, ${ }^{14}$ we define best response from every possible posterior belief.

\footnotetext{
${ }^{14}$ This follows from equation (1), which implies that $Z$ has uniformly bounded drift and a volatility that is uniformly bounded below.
} 
DEFINITION 2: In our setting, we define equilibrium to be a pair of strategies $(a, s) \in \mathcal{A} \times \mathcal{S}$ such that, for all $p \in(0,1)$ :

(i) Worker Optimality: The control solves the worker's problem, i.e.,

$$
a \in \underset{\hat{a} \in \mathcal{A}}{\operatorname{argmax}} v(p \mid \hat{a}, s) .
$$

(ii) Instantaneous Sequential Rationality: If $s=\infty$ in a neighborhood of $p$, then the distance between $a_{\Delta}^{*}(p \mid a, s)$ and $a(p)$ tends to zero as $\Delta$ does, where, for any $\Delta>0$,

$$
\begin{aligned}
& a_{\Delta}^{*}(p \mid a, s) \\
& :=\underset{\hat{a} \in[\underline{a}, \bar{a}]}{\operatorname{argmax}}\left\{\left(1-e^{-\Delta}\right)+e^{-\Delta} E\left[v\left(P_{\Delta} \mid a, s\right) \mid P_{0}=p, A_{\tau}=\hat{a} \forall \tau \in[0, \Delta)\right]\right\} .
\end{aligned}
$$

(iii) Firm Optimality: The stopping rule solves the firm's problem, i.e.,

$$
s \in \underset{\hat{s} \in \mathcal{S}}{\operatorname{argmax}} \pi(p \mid a, \hat{s}) .
$$

An unrefined equilibrium is a pair $(a, s) \in \mathcal{A} \times \mathcal{S}$ satisfying conditions $(i)$ and (iii) (but not necessarily (ii)) above.

Worker optimality and firm optimality are standard, requiring that the strategy profile constitute a Nash equilibrium in each subgame. The requirement of instantaneous sequential rationality is the only nonstandard one, its form being tailored for continuous time. We want to require that the worker is behaving optimally at every history. In a discrete time analogue of our game, a currently hired worker knows his action will be able to affect the public belief until the next period, and so he best responds to that knowledge; instantaneous sequential rationality recovers this intuition, having a worker respond as though he will at least retain his job for the next instant. As sequential rationality does in discrete time dynamic games, the instantaneous sequential rationality refinement constrains equilibrium play by limiting off path behavior to be credible.

While our focus is on characterizing the unique equilibrium of our game (in Theorem 1), we also characterize the play associated with all unrefined equilibria (Proposition 2), and show that the former is a Pareto optimum within the latter.

\section{Main Results}

The relationship in our model is a simple one. Neither player possesses any private information about match quality, nor about past behavior. The firm benefits (both myopically and from additional information) from having the worker choose a high action, while the worker suffers no direct cost from providing this benefit. Therefore, if the firm could commit to an action-contingent firing strategy, the worker could optimally choose the high action, and the firm would solve a simple 
optimal stopping problem given this worker behavior. ${ }^{15}$ However, as the following proposition clarifies, even with no direct conflict of interest within a given period, a conflict arises in equilibrium: the firm's equilibrium profit is as though the worker always chooses the firm's least favorite action.

PROPOSITION 1: In any equilibrium $(a, s)$, the firm's value function is $\pi(\cdot \mid a, s)=\sup _{\tilde{s} \in \mathcal{S}} \pi(\cdot \mid \underline{a}, \tilde{s})$.

Let us now sketch a proof of the proposition, formally proven (as are all other formal results) in the Appendix.

We first show that the given value, $\sup _{\tilde{s} \in \mathcal{S}} \pi(\cdot \mid \underline{a}, \tilde{s})$, is an upper bound on the firm's equilibrium value. Recall that employing the worker is profitable if and only if he is well-matched to the job. Given this, the firm is certain to fire the worker if they become sufficiently pessimistic about their match quality. Firing will therefore happen in the future with positive probability. The worker's value function is then, at every belief $P_{t}$ for which the firm is sure to retain him, strictly lower than his static wage. As

$$
v\left(P_{t}\right) d t \approx \underbrace{1 d t}_{\text {Today's wage }}+(1-d t) \underbrace{E\left[d v\left(P_{t}\right)\right]}_{\text {Value increment }},
$$

it must be that $E\left[d v\left(P_{t}\right)\right]<0$. Beliefs being a martingale, this means that $v$ is strictly concave around such a belief. Consequently, the worker does not want to generate mean-preserving spreads in beliefs, i.e., he wishes to slow the flow of information. Therefore, at any belief at which the firm's value is strictly positive, the worker must choose the low action $(\underline{a})$. While the firm may have many optimal responses to the worker's equilibrium strategy, one such optimal stopping rule is the strategy $s_{0}$, which entails firing the agent as soon as his belief reaches $\pi^{-1}(0)$. Pairing this firm strategy with the worker's equilibrium strategy, $a$, then generates precisely the same path of play as does $\left(\underline{a}, s_{0}\right)$ : the worker chooses the low action until beliefs reach $\pi^{-1}(0)$, and then he is fired. In particular, $\pi\left(a, s_{0}\right)$ and $\pi\left(\underline{a}, s_{0}\right)$ are equal. But the former is a lower bound (by firm incentives) on the firm's equilibrium payoff, and the latter is exactly $\sup _{\tilde{s} \in \mathcal{S}} \pi(\cdot \mid \underline{a}, \tilde{s})$, delivering the bound.

Conversely, the firm's equilibrium value is weakly higher than its hypothetical value if the worker were to choose the low action everywhere. Indeed, any other action generates higher flow profit and better information, both of which benefit the firm.

Proposition 1 says that the ongoing nature of a worker-firm relationship entails a severe agency problem. However secure the worker's job is today, he can never enjoy absolute job security in the future, as there is always a positive probability of bad news forcing the firm's hand. ${ }^{16}$ This future contingency contaminates the

\footnotetext{
${ }^{15}$ A "forcing" strategy of firing the worker the instant he deviates to choose $A_{t} \neq \bar{a}$ would make this incentive compatible for the worker.

${ }^{16}$ If our firm could commit to retain the worker irrespective of performance, the worker could optimally choose $\bar{a}$; this arrangement would benefit the firm for some parameter values. The benefit of term limits, as proposed
} 
relationship today. The ability to fire the worker is a valuable option for the firm, who may learn ex post that the worker is not profitable to retain, but this threat comes at a cost, hindering productivity on the job.

By narrowing the possible equilibrium payoffs for the firm, Proposition 1 provides some useful information on the form of equilibrium behavior. There is some simple cutoff rule $s^{\bar{p}}$, which is a best response to the fixed control strategy $\underline{a}$, and one can further deduce that $s^{\bar{p}}$ is a firm best response to the equilibrium control $a$. However, $\left(\underline{a}, s^{\bar{p}}\right)$ is not generally an equilibrium. To see why, consider the incentives of a worker who finds himself facing $s^{\bar{p}}$ and yet employed at belief $p<\bar{p}$. His job is so insecure that he has nothing to lose. Being (instantaneously) sequentially rational, he will seek to maximize the probability that the posterior belief exceeds $\bar{p}$ the following instant. His best possible chance of escaping the imminent firing threat is to choose an action that speeds up learning, i.e., induces the largest possible variation in posterior beliefs. This is achieved by choosing the highest action at such beliefs. Consider the implications of this behavior if the high action is much more productive than the low one. Firm incentives unravel: given high action in the firing region, the firm has a strict incentive to lower its cutoff.

The argument of the above paragraph teaches us two things. First, the profile $\left(\underline{a}, s^{\bar{p}}\right)$ cannot be an equilibrium because of worker incentives below the cutoff. Second, if the productivity gap between actions is high enough $(\bar{a} \bar{p}-c>0)$, then firm incentives just below the putative cutoff preclude $s^{\bar{p}}$ being used at all in equilibrium. ${ }^{17}$

If higher actions are sufficiently valuable to the firm, then a simple cutoff strategy cannot be used in equilibrium; and a similar argument will show that the firm cannot use any deterministic firing rule in equilibrium in this case. However, given the low action, the firm is barely willing to retain the worker at $\bar{p}$. How then, can it be induced to retain the worker (even stochastically) when even more pessimistic? The worker must willingly choose higher actions over this mixing region.

Our main theorem, a complete characterization of the unique equilibrium, formalizes the above intuition.

THEOREM 1: There is a unique equilibrium $\left(a^{*}, s^{*}\right)$. In equilibrium, there are two cutoffs $0<\underline{p} \leq \bar{p}<c / \underline{a}$ such that:

(i) Above $\bar{p}$, the firm never fires the worker, who in turn chooses the lowest action.

(ii) Between $p$ and $\bar{p}$, the firm mixes, the worker produces zero expected flow profit $t^{18}$ for the firm, and the worker's value function is affine. As $p$ increases from $\underline{p}$ to $\bar{p}, s^{*}(p)$ decreases continuously from $\infty$ to some $\underline{s}>0$.

\footnotetext{
by Aghion and Jackson (2016), has a similar intuition. Tying the principal's hands limits the agent's scope for inefficient career-concerned behavior.

${ }^{17}$ In the complementary case, when $\bar{a} \bar{p}-c \leq 0$, the firm can use $s^{\bar{p}}$ in equilibrium, with the worker's behavior switching at the same cutoff.

${ }^{18}$ That is, his action at such a belief $p$ satisfies $a^{*}(p) p-c=0$.
} 
(iii) Below p, the firm immediately fires the worker.

When higher actions are sufficiently valuable, the middle region is nondegenerate. 19

We prove the theorem in the Appendix, but it may be instructive to sketch the argument that rules out all other candidate equilibria.

Letting $\bar{p}$ be the cutoff belief at which the firm would fire if the worker were always to choose the low action, Proposition 1 says that the firm has strictly positive continuation value to the right of $\bar{p}$ and zero value to the left of it. Zero value implies that the firm's flow profit $a(p) p-c$ must be nonpositive at every belief $p \leq \bar{p}$.

Now, let $I$ be any maximal interval of beliefs below $\bar{p}$ at which the agent enjoys a strictly positive continuation value. In particular, the firm's firing rate is finite over $I$. Starting from $P_{t} \in I$, the firm's continuation profit at $P_{t+d t} \in I$ is certain to be zero, and the firm is still willing to retain the worker. This rules out strictly negative flow profits: the firm earns zero flow profits over $I$. That is, the worker chooses action $a(p)=c / p$ there. Worker optimality then requires that he be indifferent to creating information, to be willing to choose an interior action: $\left.v\right|_{I}$ must be affine. But then, $I$ must have $\bar{p}$ as one of its endpoints. Otherwise, the agent would have a zero continuation value at either endpoint, and so (by affinity) could not obtain a strictly positive value over $I$.

The above argument shows that there is some $p \leq \bar{p}$ such that the firm immediately fires at beliefs below $\underline{p}$ but not at beliefs in $(\underline{p}, \bar{p}]$, and the firm receives zero flow profit over $(p, \bar{p}]$. Next, instantaneous sequential rationality says that the worker will choose the high action to the left of $\underline{p}$, in a last-ditch effort to escape the firing region. ${ }^{20}$ By firm optimality, the high action must be enough to secure nonnegative flow profit over $(\underline{p}, \bar{p}]$ but not enough to induce strictly positive flow profit to the left of $p$; these features pin down the cutoff $p$ exactly.

Notice that, as shown in Figure 3, both players' behavior is discontinuous in the unique equilibrium. Discontinuity of the firm's retention decision is interpretable: employees with very similar performance histories can experience markedly different treatment from their employers in equilibrium.

The region $(\underline{p}, \bar{p})$ can be thought of as a probationary period. Here, the employee works harder in the face of an imminent threat of losing his job. Should enough good news accrue, the employee is "promoted" to a region above $\bar{p}$, where he faces no such imminent threat. The discontinuity of the worker's behavior is broadly consistent with an observation made by Engellandt and Riphahn (2005), Ichino and Riphahn (2005), and Jacob (2013): employees' productive effort systematically falls as a probationary period ends.

\footnotetext{
${ }^{19}$ More precisely, $\underline{p}<\bar{p}$ if and only if $\bar{a}>\underline{a}+((\underline{a}-c) / \delta)$, where $\delta=(1 / 2)(\sqrt{1+(1 /(8 \underline{a}))}-1)>0$.

${ }^{20}$ A similar mechanism emerges (on-path) in Varas (2019), wherein a manager takes a risky short-termist action at the brink of dismissal. See also Anderson and Cabral (2007) for a similar appeal (in an R\&D competition model) to endogenously arising preferences over volatility.
} 


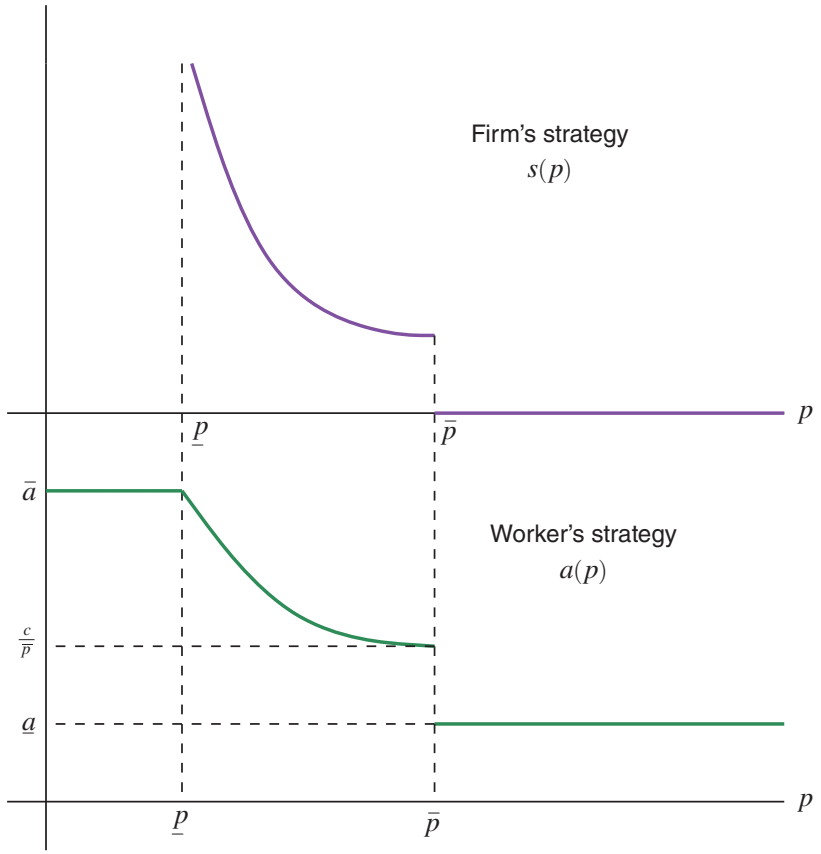

Figure 3. Players' Strategies

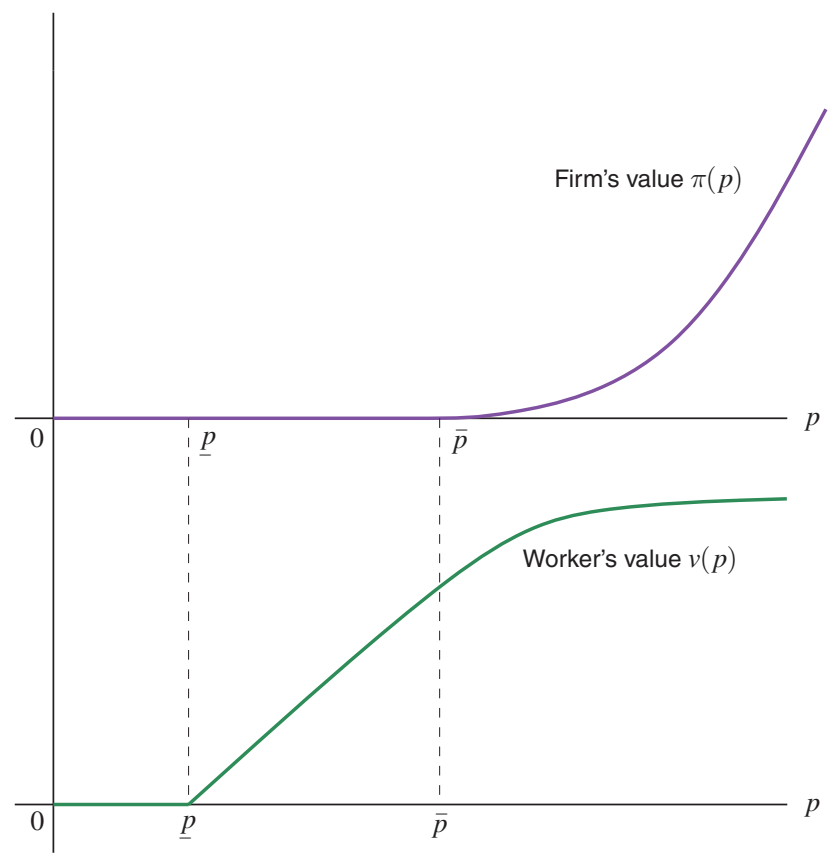

Figure 4. Players' Value Functions 
While our instantaneous sequential rationality refinement delivers a unique equilibrium, the analysis leading to Theorem 1 still characterizes the equilibrium play ranging across all unrefined equilibria. ${ }^{21}$

PROPOSITION 2: For every $p_{L} \in[\underline{p}, \bar{p}]$, there is an unrefined equilibrium in which:

(i) Above $\bar{p}$, the firm never fires the worker, who in turn chooses the low action.

(ii) Between $p_{L}$ and $\bar{p}$, the firm mixes, the worker produces zero expected flow profit for the firm, and the worker's value function is affine. As $p$ increases from $p_{L}$ to $\bar{p}$, the firm's firing rate decreases continuously from $\infty$ to some $\underline{s}>0$.

(iii) Below $p_{L}$, the firm immediately fires the worker.

Moreover, every unrefined equilibrium is of the above form, and the unique (refined) equilibrium is Pareto optimal within the class.

\section{A. Job Security and Productivity}

In our model, a worker's continuation value $V_{t}$ is his expected discounted future employment, i.e., his job security. If he chooses $A_{t}$ while believed to be well-matched to the firm with probability $P_{t}$, then his current expected flow profitability, i.e., his productivity, is $A_{t} P_{t}-c$. Therefore, in equilibrium, belief about match quality is a common determinant of both job security and productivity. Given this, a relationship between the latter two follows directly from the theorem.

COROLLARY 1: Consider an equilibrium $\left(a^{*}, s^{*}\right)$, and let $v^{*}$ be the associated worker value function. For any level $v \in(0,1)$ of job security, there is a unique belief $p(v) \in(0,1)$ such that $v^{*}(p(v))=v$. Let $F(v):=p(v) a^{*}(p(v))-c$, the worker's current productivity at this level of job security. Then there exists $\hat{v} \in[0,1)$ such that:

(i) On $(0, \hat{v}), F$ is zero.

(ii) On $(\hat{v}, 1), F$ is strictly convex and strictly increases from negative to positive.

When higher actions are sufficiently valuable (as described in footnote 19), $\hat{v}>0$.

When the worker's job is very insecure (below $\hat{v}$ ), he is productive enough to barely be worth employing for the moment. A more secure worker chooses the lowest

\footnotetext{
${ }^{21}$ A proof of this proposition is a straightforward modification of the proof of Theorem 1; we therefore omit it. One follows the same line of argument, without an appeal to Lemmas 12 and 13, and replacing $\underline{p}$ with $p_{L}$ in Lemmas 14 and 15.
} 


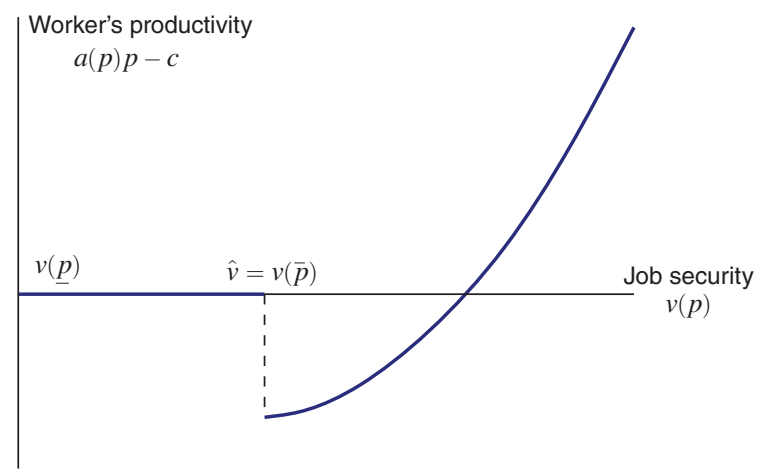

Figure 5. Observed Relationship between Job Security and Productivity

action, but what are the productivity implications? A very secure worker is productive, on average, because he is likely to be well-matched to the firm. A moderately secure worker (with job security barely above $\hat{v}$ ) loses money for the firm today: he is rationally retained only for option value reasons.

Thus, equilibrium play in our model generates a U-shaped pattern: a worker is least productive when his job is moderately secure. ${ }^{22}$

\section{Dynamic Games in Continuous Time}

Beyond studying the relationship between job security and productivity, we hope that our paper contributes more broadly to the study of dynamic games in continuous time. First, we provide a refinement, instantaneous sequential rationality, which mirrors discrete time intuitions and yet has refining power in our continuous time application (and hopefully elsewhere). Second, we extend the direct applicability of known methods from continuous time dynamic optimization to settings in which multiple (long-lived, strategic, "large") players interact and may behave discontinuously.

\section{A. A Sequential Rationality Refinement}

Our environment gives cause to consider players' optimal behavior at contingencies they never expect to reach in equilibrium, i.e., off the path of play. Indeed, consider a belief at which the firm plans to immediately fire the worker. How should the worker behave, if he finds himself mysteriously employed at such a belief?

In a discrete-time setting, the worker would take his current employment as given, and would seek to maximize his prospects from the next period onward. In continuous time, "the next period" will arrive immediately. Thus, the worker is indifferent: his choice is inconsequential to his future. This is unsatisfactory if one

\footnotetext{
${ }^{22}$ The assumption that the firm finds it profitable to employ the well-matched worker even when he is choosing $\underline{a}$ plays an important role here. Without this assumption, the unique equilibrium would entail worker productivity of zero, as in the model of Garfagnini (2011).
} 
wants to interpret the continuous time model (as we do) as simply a mathematically convenient approximation of a discrete time model.

We offer a refinement of MPE for continuous time dynamic games, termed instantaneous sequential rationality, whose intuition is drawn from sequential rationality in discrete time. Analogous to a discrete time setting, we assume that a player responds optimally, taking for granted that his current decision will be relevant for the next instant. More formally, at an off-path state, we consider the worker's hypothetical optimal action $a_{\Delta}$ if he were sure to retain his job for the next $\Delta>0$ units of time. Our refinement then requires his behavior be a limit of $a_{\Delta}$ as $\Delta$ vanishes.

While the "period length" implicit in our refinement is small, there are behavioral implications. In our model, absent instantaneous sequential rationality, Proposition 2 delivers a continuum family of equilibria with distinct on-path behavior. Our refinement yields a unique selection (of a Pareto optimal member) from this family.

Ours is not the first paper to invoke specific refinements in a continuous-time game to reproduce intuitions from a discrete-time analogue. For instance, see Ortner (2019). While we do not presently develop a general theory of instantaneous sequential rationality, we are hopeful that some incarnation of it can be fruitful in future applications as well.

\section{B. Dynamic Optimization Methods with Multiple Players}

Stochastic control in continuous time yields powerful methods that have been applied to dynamic games. ${ }^{23}$ Two prevalent approaches to solving decision problems in continuous time involve the Hamilton-Jacobi-Bellman (HJB) equation associated with a forward-looking player's incentives.

The first is the verification approach, which focuses on sufficient conditions for a given candidate policy to be optimal for a decision-maker. ${ }^{24}$ The second is to show that the decision-maker's value function necessarily satisfies smoothness properties and derive necessary conditions of optimal play that follow from the HJB. In The Art of Smooth Pasting, Dixit (1993) heuristically derives optimal policies in many dynamic optimization problems in this way. As described in Strulovici and Szydlowski (2015), value functions will exhibit some smoothness and HJB equations will be satisfied as long as the underlying optimization problem is sufficiently smooth.

The most important limitation of the above methods in strategic situations is that one player's behavior - and therefore the environment faced by the other optimizing player-may be discontinuous in equilibrium. Theorem 1 shows that this happens in our game. Given this complication, one must extend existing tools to apply either of the two approaches. On intervals (of the space of beliefs) where players'

\footnotetext{
${ }^{23}$ See, for example, Board and Meyer-ter-Vehn (2013); Bohren (2019); Cisternas (2018b); Daley and Green (2012); Daley and Green (2020); Dilmé (2017); Kolb (2015, 2019); Orlov, Skrzypacz, and Zryumov (2018); Varas (2019); Varas, Marinovic, and Skrzypacz (2018); and Chavez (2019).

${ }^{24}$ The analyst heuristically derives a candidate policy for the decision maker, and with it a candidate value function. A verification theorem then says: if the value function is sufficiently smooth and the candidates satisfy the HJB, then the candidates are in fact optimal. For example, see Pham (2009) for optimal control verification theorems and Øksendal (2003) for an optimal stopping verification theorem.
} 
behavior is Lipschitz continuous, nothing new is required to show that value functions are $C^{2}$ and HJB equations are satisfied. At beliefs where our firm changes its behavior discontinuously, but the firing rate is locally bounded, we derive a smooth pasting condition for the worker. The argument applies the viscosity approach (see Crandall, Ishii, and Lions (1992), for instance) to stochastic optimal control. ${ }^{25}$ With piecewise $C^{2}$ value functions and smooth pasting in hand, we are able to derive useful necessary conditions toward characterizing equilibrium in our setting.

\section{Extensions}

Our model is a stylized one, but it is also flexible. In this section, we consider some extensions of our model and briefly describe how the worker-firm relationship is affected.

\section{A. Richer Worker Controls}

For ease of exposition, our leading model consists of a one-dimensional action for the worker to take at each time. A reasonable alternative, however, is for a worker to make a broader, multidimensional decision about how to spend his time, somewhat analogous to Holmström and Milgrom (1991).

To generalize our leading model, suppose the worker chooses $A_{t} \in \Sigma$, where $\Sigma$ is a compact convex subset of some Euclidean space. Action process $\left\{A_{t}\right\}_{t}$ induces an accrued payoff process satisfying

$$
d X_{t}=\left[\beta\left(A_{t}\right)+\mu\left(A_{t}\right) \theta-c\right] d t+\sigma\left(A_{t}\right) d B_{t},
$$

where $\beta, \mu, \sigma: \Sigma \rightarrow \mathbb{R}$ are all continuous functions, and $\mu$ and $\sigma$ are strictly positive.

We make the following assumptions on the environment.

\section{ASSUMPTION 1:}

(i) There is a unique $\underline{a}[$ resp. $\bar{a}]$ in $\Sigma$ that minimizes $[$ maximizes $] \mu(\cdot) / \sigma(\cdot)$. There is a unique $a_{\$}^{0}\left[\right.$ resp. $\left.a_{\$}^{1}\right]$ in $\Sigma$ that maximizes $\beta(\cdot)$ [maximizes $\beta(\cdot)+\mu(\cdot)]$.

(ii) $a_{\$}^{0}=a_{\$}^{1} \equiv a_{\$}$.

(iii) $a_{\$} \neq \underline{a}$.

We do not see the first part of the assumption as economically substantive. For instance, suppose $\Sigma$ were the convex hull of finitely many extreme points and each

\footnotetext{
${ }^{25}$ Notice, in our equilibrium, the worker's value function has exactly one kink, depicted in Figure 4 at a belief where the firm's stopping rate explodes.
} 
of $\{\beta, \mu, \sigma\}$ were affine, so that actions could be interpreted as choices of how the worker will divide his time across these finitely many activities. Then, for generic choice of $\{\beta, \mu, \sigma\}$ (which are pinned down by the values they take on the extreme points), the first assumption would hold.

The second assumption is substantive but also meaningful. It will, for instance, hold if $\beta$ and $\mu$ vary weakly comonotonically, so that actions are unequivocally ordered in terms of flow productivity. The third assumption is also substantive, but we think this is the "less special" case. Indeed, in the affine case described in the previous paragraph, if the worker were choosing how to divide his time between many activities, then the opposite condition $\left(a_{\$}=\underline{a}\right)$ would be very special. We note that, for our leading model with one-dimensional action, taken literally, $a_{\$}$ would be equal to $\underline{a}$ if myopically profit-enhancing actions generated worse, rather than better, information.

In the bigger model sketched above, the analysis of our paper goes through with little change. As worker incentives hinge solely on information in equilibrium, Proposition 1 holds exactly, so the firm has the same profit across all equilibria. Similarly, instantaneous sequential rationality pins down the worker's behavior off-path, and a three-region structure analogous to Theorem 1 obtains. The agent plays $\bar{a}$ in the lower region and $\underline{a}$ in the top region. Zero flow profits for the firm pins down the behavior in the middle region.

Note, however, that if $a_{\$} \neq \bar{a}$, then there can be multiple beliefs that make it simultaneously possible for the firm to receive nonpositive flow profit from $\bar{a}$ (the worker's off-path action) and zero flow profit from some other action. Consequently, we might obtain multiple equilibria that differ in the left cutoff of the mixing region, and so corresponding to different worker payoffs. Note that this multiplicity is ruled out in our leading model, which has the special feature that $a_{\$}=\bar{a}$. (There is another, less economically important, multiplicity if $\Sigma$ is multidimensional, which is that the worker will typically have multiple distinct actions that keep the firm indifferent at beliefs in the mixing region.) In spite of this multiplicity, an analogue of Corollary 1 holds equally in this more general model. ${ }^{26}$

\section{B. Costly Effort}

One reasonable interpretation of a worker's actions is his choice of how hard to work. One could imagine that higher effort both increases his expected flow output and makes said output more responsive to match quality. Under this interpretation, a more natural payoff assumption would be that higher effort is costly to the worker.

If the worker suffers a private cost of exerting effort-consider the case of a linear cost - the observable implications of our model are very similar. Unrefined equilibrium still consists of a three-region structure, with firing in the pessimistic region, mutual mixing in the middle region, and low effort with no firing in the

\footnotetext{
${ }^{26}$ Just as in the baseline model, the middle region can be degenerate or non-degenerate. Here, $\hat{v}>0$ only in the latter case.
} 
optimistic region. ${ }^{27}$ That is, the family of unrefined equilibria of the game with costly effort is similar to that for our leading model: each has the same three-region structure, with the left cutoff varying across equilibria. However, with costly effort, instantaneous sequential rationality requires the worker to instead exert low effort in the pessimistic region. Therefore, every unrefined equilibrium is now path-equivalent to a refined equilibrium.

\section{Varying Wages}

Suppose the worker receives a wage that varies with his standing in the firm. If his wage $w(p)$ is weakly concave in belief $p$ (for example, it could be linear as in Holmström 1999), then our main results again go through. ${ }^{28}$

This clarifies that ours is a model of job insecurity paired with low-powered incentives. In particular, the logic of Proposition 1 relies on the worker facing job insecurity following poor performance and no steep reward following good performance.

Kostadinov and Kuvalekar (forthcoming) analyze a related setting (albeit in discrete time and with costly effort) with time-varying discretionary wages. Their focus is on constrained-surplus-maximizing, non-Markovian equilibrium. ${ }^{29}$ The differing predictions of our model and that of Kostadinov and Kuvalekar (forthcoming) are substantive. In particular, in contrast to our Theorem 1, their agent takes higher actions in that model as the players become optimistic - productivity is monotonically increasing in job security.

\section{Promotion}

One way of providing incentives in an ongoing worker-firm relationship is through promotions. Our model can accommodate this.

Suppose there is an exogenous cutoff $\hat{p}$ above which the worker has exogenous continuation value $\hat{w}$ upon reaching $\hat{p}$. For instance, the worker could be tenured with wage $\hat{w}$. If $\hat{w} \leq w$, then one can show that the structure of our equlibrium in Theorem 1 persists: even the lure of tenure cannot incentivize a currently untenured worker to choose the high action.

More interesting is the case where $\hat{w}>w$ : a worker is unambiguously better off once promoted. We can construct an equilibrium using similar reasoning to that of the baseline model. As the worker nears the promotion threshold, the possibility of promotion serves as a high-powered incentive: he chooses the high action, aiming to accelerate this reward. One can show that this equilibrium will exhibit a four-regime structure, as in Figure 6, with a new high-action regime at the most

\footnotetext{
${ }^{27}$ In this model, for equilibrium to exist, the worker must be allowed to mix rather than simply choosing an interior effort level. In the middle region, he mixes over $\{\underline{a}, \bar{a}\}$ to give the firm a flow payoff of zero.

${ }^{28}$ It would then also be natural to allow for the firm's cost of retaining the worker to depend on expected match quality, e.g., if $c(\cdot)=w(\cdot)$. In this case, we would also want to assume that $\underline{a} p-c(p)$ is strictly increasing in $p$, so that a higher expected match quality leads to higher flow profit.

${ }^{29}$ Although arbitrary history dependence is, in principle, allowed, it is without loss of optimality for payments to condition only on the current beliefs about match quality and on behavior in the most recent period. This result reflects a standard principle for relational contracts with transferable utility (e.g., Levin 2003).
} 


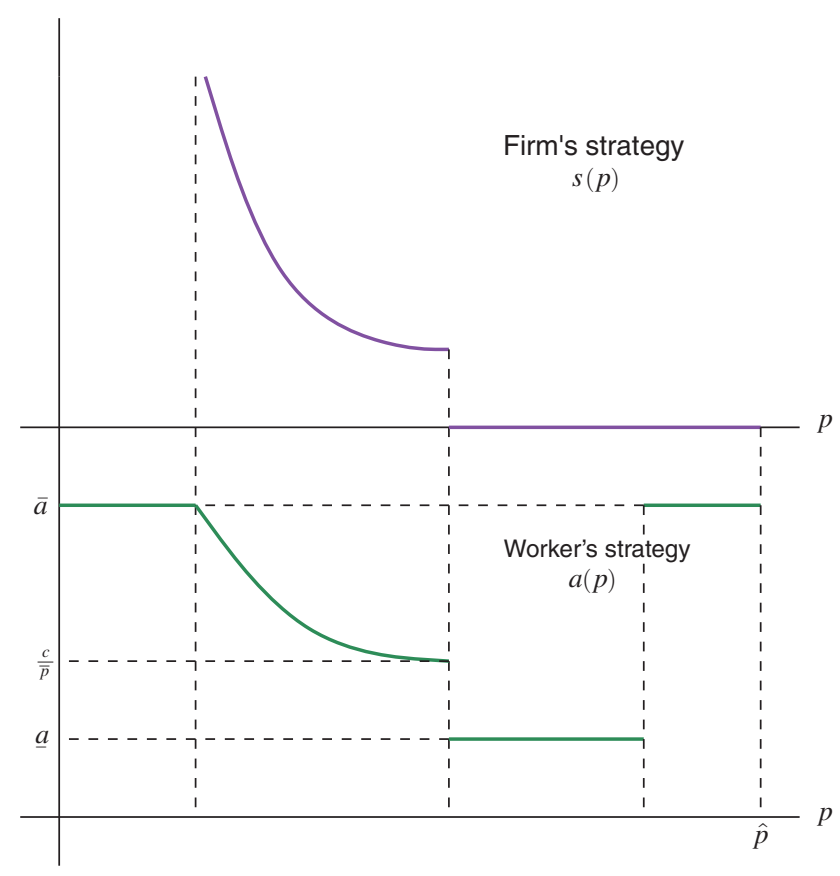

Figure 6. Players' Strategies with Promotion at $\hat{p}$ IF $\hat{w}>w$

optimistic beliefs. Just as in our leading model, the region with firm mixing may be degenerate.

\section{E. Beyond Markovian Play}

It is well known that modeling non-Markovian play in continuous-time settings with observable behavior brings conceptual subtleties, in particular concerning how one defines a player's strategy space, and how one maps a strategy profile into a realized path of play. ${ }^{30}$ Hence, one may be required to explicitly model continuous-time play as a limit of discrete-time play (as in Simon and Stinchcombe (1989)), possibly foregoing much of the analytical tractability of continuous-time methods. In part for this reason, we follow the literature in focusing our attention on Markovian play.

While rigorously formalizing continuous time strategies is beyond the scope of this paper, let us heuristically demonstrate how, it seems, relational incentives can be profit-enhancing for our modeled employment relationship. To see this, we restrict to the case (see footnote 19) that the mixing region from Theorem 1 is nondegenerate. Consider a two-phase relationship, as depicted in Figure 7. In both phases, players

\footnotetext{
${ }^{30}$ This subtlety can be sidestepped in settings with imperfect public monitoring (e.g., Sannikov 2007), by focusing attention on equilibrium in public strategies. There, the feasible paths of public outcomes not varying with players' behavior, defining players' strategies and the induced law for outcomes is more straightforward.
} 


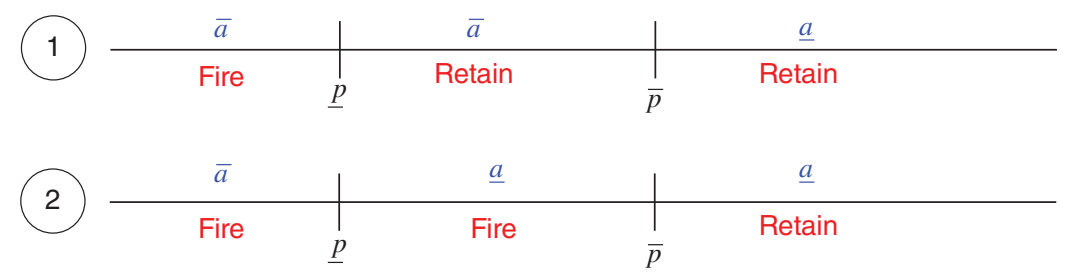

Figure 7. Non-Markovian "Strategies"

behave exactly as in the unique equilibrium of Theorem 1, except in the mixing region. In phase 1 , at beliefs in $(p, \bar{p}]$, the firm retains the worker $(s=0)$ with certainty, and the worker chooses $\bar{a}$. As soon as anybody deviates from the prescribed play of phase 1 , the players immediately and permanently move to phase 2 . In this phase, over $(\underline{p}, \bar{p}]$, the firm fires the worker immediately, and the worker chooses $\underline{a}$. If credible, this play makes the firm strictly better off than does our Markovian equilibrium. In particular, in contrast to Proposition 1, the firm derives a strictly positive profit from every prior belief above $\underline{p}$.

Given Proposition 2, one easily sees that phase 2 describes an unrefined equilibrium. To verify firm incentives in phase 1 , notice that continuation profits are always nonnegative, so that there is never an incentive to deviate to fire. Moreover, at beliefs below $p$, retaining the worker would result in a negative flow payoff, followed by immediate reversion to phase 2 , yielding negative total profit. We now turn to the worker. Outside of $(\underline{p}, \bar{p}]$, his incentives are qualitatively similar to in our Markovian equilibrium. In $(\underline{p}, \bar{p}]$, the worker has a strictly positive continuation value, while any deviation leads to immediate termination. Thus, this code of play is self-enforcing.

Recall that, in line with Proposition 2, every unrefined equilibrium is profit-equivalent to our refined equilibrium. Thus, without instantaneous sequential rationality, it appears that relational incentives can be strictly profit-enhancing. However, the above construction fails this refinement: middle-region play in the Markovian phase 2 is not instantaneously sequentially rational. We are at this point unsure of whether the firm can profit from relational incentives while respecting this additional constraint. We view formalization of a model of non-Markovian equilibrium for this game, and the resolution of this question about life inside our firm, as an exciting avenue for future work.

\section{Concluding Remarks}

In this article, we have explored the relationship between job security and productivity in organizations with low-powered incentives and limited commitment. The potential for a U-shaped relationship has imprints of two quite different managerial styles. The "drive in fear" approach of Intel's Grove suggests that appropriately deployed threats can help combat complacency in the workplace. This principle is reflected at moderate beliefs in our model, where the threat of firing is nontrivial and the worker takes higher actions. The "drive out fear" school of 
Deming points to a lurking hazard of job insecurity: workers will fear taking riskier actions, and this fear distorts organizational decision-making. This force describes optimistic beliefs in our model, where the worker takes inefficiently low actions for fear of his future employment.

Beyond the applied analysis of our model, we hope that our paper might be of broader interest for the study of dynamic games in continuous time. In particular, our "instantaneous sequential rationality" refinement mirrors discrete-time intuitions and selects a unique equilibrium in our model. Moreover, the viscosity approach adapted herein seems especially convenient for proving smooth-pasting properties in dynamic games for which individual incentives yield discontinuous play. We are hopeful that both of these contributions prove useful in analyzing other dynamic interactions of economic interest.

\section{Mathematical ApPendix}

\section{A. Technical Preliminaries}

Players' equilibrium value functions might be wild. In particular, as one player's behavior might change discontinuously with beliefs-and may be required to by his/her own best response property-neither value function is known to be the optimal value function to a well-behaved decision problem. Even so, one can obtain various regularity properties, which will be helpful for bringing the toolset of stochastic calculus to bear in understanding our setting.

LEMMA 1: Given any $(a, s) \in \mathcal{A} \times \mathcal{S}$, the value functions $\pi(\cdot \mid a, s), v(\cdot \mid a, s)$ and the optimal value functions $\sup _{\tilde{s} \in \mathcal{S}} \pi(\cdot \mid a, \tilde{s}), \sup _{\tilde{a} \in \mathcal{A}} v(\cdot \mid \tilde{a}, s)$ are continuous on $(0,1)$.

\section{PROOF:}

Given $0<q<p<q+\epsilon<1$, let $\left\{P_{t}^{p}\right\}_{t}$ denote the belief process starting at $P_{0}=p$, and define the $P^{p}$-measurable stopping time $\tau_{q, \epsilon}^{p}:=\inf \left\{t \geq 0: P_{t}^{p} \notin(q, q+\epsilon)\right\}$.

To begin with, we prove the following claim: For any $s \in \mathcal{S}$ and $q \in(0,1)$, there are real constants $K, L>0$ and $\epsilon>0$ such that

$$
|v(p \mid a, s)-v(q \mid a, s)|,|\pi(p \mid a, s)-\pi(q \mid a, s)| \leq K E_{a}\left(\tau_{q, \epsilon}^{p}\right)+L \frac{p-q}{\epsilon}
$$

for all $a \in \mathcal{A}$.

Toward the claim, fix $(a, s) \in \mathcal{A} \times \mathcal{S}$ and $q \in(0,1)$, and let $g \in\{v(\cdot \mid a, s), \pi(\cdot \mid a, s)\}$. Then there is a bounded, piecewise Lipschitz function $f:[0,1] \rightarrow \mathbb{R}$ such that ${ }^{31}$

$$
\begin{aligned}
& g(p)=E_{a}\left[\int_{0}^{\infty} e^{-t} e^{-\int_{0}^{t} s(P \tau) d \tau} f\left(P_{t}\right) d t \mid P_{0}=p\right] . \\
& { }^{31} \text { Indeed } f(p)=1 \text { if } g=v(\cdot \mid a, s) \text {, and } f(p)=a(p) p-c \text { if } g=\pi(\cdot \mid a, s) .
\end{aligned}
$$


For now, focus on the case of $s(q)<\infty$. Let $\epsilon \in(0,1-q)$ be small enough to ensure $\left.s\right|_{(q, q+\epsilon)}$ is bounded above by a constant $\bar{s} \in \mathbb{R}_{+}$. Let $\beta_{t}^{p}:=e^{-t} e^{-\int_{0}^{t} s\left(P_{\hat{t}}^{p}\right) d \hat{t}}$ and $\tau^{p}:=\tau_{q, \epsilon}^{p}$. Then, for any $p \in(q, q+\epsilon)$ :

$$
\begin{aligned}
|g(p)-g(q)|= & \left|E_{a}\left[\int_{0}^{\infty} \beta_{t}^{p} f\left(P_{t}^{p}\right) d t\right]-g(q)\right| \\
= & \left|E_{a}\left[\int_{0}^{\tau^{p}} \beta_{t}^{p} f\left(P_{t}^{p}\right) d t+\beta_{\tau^{p}}^{p} g\left(P_{\tau^{p}}^{p}\right)\right]-g(q)\right| \\
= & \left|E_{a}\left\{\int_{0}^{\tau^{p}} \beta_{t}^{p} f\left(P_{t}^{p}\right) d t-\left(1-\beta_{\tau^{p}}^{p}\right) g(q)+\beta_{\tau^{p}}^{p}\left[g\left(P_{\tau^{p}}^{p}\right)-g(q)\right]\right\}\right| \\
= & \left|E_{a}\left\{\int_{0}^{\tau^{p}} \beta_{t}^{p}\left[f\left(P_{t}^{p}\right)-\left(1+s\left(P_{t}\right)\right) g(q)\right] d t+\beta_{\tau^{p}}^{p}\left[g\left(P_{\tau^{p}}^{p}\right)-g(q)\right]\right\}\right| \\
\leq & E_{a} \int_{0}^{\tau^{p}} \beta_{t}^{p}\left|f\left(P_{t}^{p}\right)-\left[1+s\left(P_{t}^{p}\right)\right] g(q)\right| d t+E_{a} \beta_{\tau^{p}}^{p}\left|g\left(P_{\tau^{p}}^{p}\right)-g(q)\right| \\
\leq & E_{a} \int_{0}^{\tau^{p}}\left|f\left(P_{t}^{p}\right)-\left[1+s\left(P_{t}^{p}\right)\right] g(q)\right| d t \\
& \quad+|g(q+\epsilon)-g(q)| \operatorname{Pr}_{a}\left\{P_{t}^{p}=q+\epsilon\right\} .
\end{aligned}
$$

So let $K:=\|f\|_{\infty}+(1+\bar{s})|g(q)|$ and $L:=|g(q+\epsilon)-g(q)|$. Then, $|g(p)-g(q)| \leq K E_{a}\left(\tau^{p}\right)+L((p-q) / \epsilon)$, as $P_{t}^{p}$ is a martingale.

Now consider the case of $s(q)=\infty$. Let $\hat{\tau}^{p}:=\inf \left\{t \geq 0: P_{t}^{p}=q\right\}$. Then

$$
\begin{aligned}
|g(p)-g(q)| & =\left|E_{a}\left[\int_{0}^{\infty} \beta_{t}^{p} f\left(P_{t}^{p}\right) d t\right]-g(q)\right| \\
& =\left|E_{a}\left[\int_{0}^{\hat{\tau}^{p}} \beta_{t}^{p} f\left(P_{t}^{p}\right) d t+\beta_{\hat{\tau}^{p}}^{p} g(q)\right]-g(q)\right| \\
& =\left|E_{a}\left[\int_{0}^{\hat{\tau}^{p}} \beta_{t}^{p} f\left(P_{t}^{p}\right) d t\right]\right| \\
& \leq E_{a} \int_{0}^{\hat{\tau}^{p}}\left|f\left(P_{t}^{p}\right)\right| d t,
\end{aligned}
$$

so that letting $K:=\|f\|_{\infty}$ (with any $L, \epsilon$ ) completes the proof of the claim.

Now, standard dynamic programming arguments, together with the martingale property of beliefs, tell us that $h(p):=\sup _{a \in \mathcal{A}} E_{a}\left(\tau_{q, \epsilon}^{p}\right)$ is concave in $p$, so that $\sup _{a \in \mathcal{A}} E_{a}\left(\tau_{q, \epsilon}^{p}\right)=E_{\underline{a}}\left(\tau_{q, \epsilon}^{p}\right)$. 
Therefore, we can find constants $K, L, \epsilon>0$ such that, for every $p \in(q, q+\epsilon)$, the (control-independent) number,

$$
M^{p}=K E_{\underline{a}}\left(\tau_{q, \epsilon}^{p}\right)+L \frac{p-q}{\epsilon},
$$

is a uniform upper bound for $\{|v(p \mid a, s)-v(q \mid a, s)|, \mid \pi(p \mid a, s)-$ $\pi(q \mid a, s) \mid: a \in \mathcal{A}\}$.

Finally, $M^{p} \rightarrow 0$ as $p \rightarrow q$ because $E_{\underline{a}}\left(\tau_{q, \epsilon}^{p}\right)$ does.

The above argument implies that $v(\cdot \mid a, s), \pi(\cdot \mid a, s)$ are right-continuous at $q$ for fixed control, and that $\sup _{a \in \mathcal{A}} v(\cdot \mid a, s)$ is right-continuous there. An identical argument shows that these functions are left-continuous there as well.

Finally, fix any $a \in \mathcal{A}$. The optimal value function $\pi=\sup _{s \in \mathcal{S}} \pi(\cdot \mid a, s)$ is a supremum of continuous functions, and so is lower-semicontinuous. Therefore, its zero set is closed, so that $s:=\left.\left.\infty\right|_{\pi^{-1}(0)} \cup 0\right|_{[0,1] \backslash \pi^{-1}(0)}$ is an element of $\mathcal{S}$. It is easy to see that $s$ is a firm best response to $a$, so that $\pi=\pi(\cdot \mid a, s)$ is continuous.

All that remains is to check continuity at an endpoint $p \in\{0,1\}$. Notice that ${ }^{32}$ for any $\epsilon, \phi \in(0,1)$, there exists $\epsilon^{\prime} \in(0, \epsilon)$ such that

$$
\inf _{a \in \mathcal{A}} E_{a}\left[\int_{0}^{\infty} e^{-t} \mathbf{1}_{\left|P_{\tau}-p\right|<\epsilon \forall \tau \in[0, t]} d t\right] \geq \phi
$$

whenever $P_{0}$ is within $\epsilon^{\prime}$ of $p$. Therefore:

- If $s(p)=\infty$, continuity ${ }^{33}$ of $s$ in a neighborhood of $p$ implies that the value functions tend to zero near $p$.

- If $s(p)<\infty$, then play is Lipschitz in a neighborhood of $p$. It follows that the limiting worker payoff is 1 and the limiting firm payoff is $a(p) p-c$.

In either case, the limiting payoff agrees with the payoff at $p$ itself by direct computation.

LEMMA 2: Fix $0 \leq p_{0}<p_{1} \leq 1$. Suppose $s \in \mathcal{S}$ is such that $\left.s\right|_{\left[p_{0}, p_{1}\right]}$ is finite-valued and Lipschitz, and $a \in \mathcal{A}$ such that $\left.a\right|_{\left[p_{0}, p_{1}\right]}$ is Lipschitz. Let $v^{*}:=\sup _{a \in \mathcal{A}} v(\cdot \mid a, s)$ and $v:=v(\cdot \mid a, s)$. Then $v^{*}, v$ are $C^{2}$ on $\left[p_{0}, p_{1}\right]^{34}$ and satisfy:

$$
\begin{aligned}
& 0=\frac{1}{2}[a(p) p(1-p)]^{2} v^{\prime \prime}(p)-[1+s(p)] v(p)+1, \\
& 0=\sup _{\hat{a} \in[\underline{a}, \bar{a}]}\left\{\frac{1}{2}[\hat{a} p(1-p)]^{2} v^{* \prime \prime}(p)-[1+s(p)] v^{*}(p)+1\right\} .
\end{aligned}
$$

\footnotetext{
${ }^{32}$ This can be seen by boundedness of $[\underline{a}, \bar{a}]$ and the law of motion (1).

${ }^{33}$ This is the only place in the paper where piecewise continuity of $s$ is required. In particular, it could be replaced with the weaker condition that $\lim _{\hat{p} \rightarrow p} s(\hat{p})=\infty$ if $p \in\{0,1\}$ and $s(p)=\infty$, with no change to our results.

${ }^{34}$ Where derivatives at $p_{0}, p_{1}$ are taken to be limits of derivatives.
} 
Moreover, if a $C^{2}$ function $f:\left[p_{0}, p_{1}\right] \rightarrow \mathbb{R}$ satisfies

$$
0=\frac{1}{2}[a(p) p(1-p)]^{2} f^{\prime \prime}(p)-[1+s(p)] f(p)+1
$$

together with the boundary conditions $\left(f\left(p_{0}\right), f\left(p_{1}\right)\right)=\left(v\left(p_{0}\right), v\left(p_{1}\right)\right)$, then $f=\left.v\right|_{\left[p_{0}, p_{1}\right]}$; similarly for $v^{*}$.

\section{PROOF:}

This follows immediately from (Krylov 2009, Chapter 1, Theorem 5). To apply that result, we note that any control $a \in \mathcal{A}$ that is optimal among all admissible controls is clearly optimal within the restricted class $\mathcal{A}$.

In our setup the firm solves an optimal stopping problem. In the terminology of the previous section, the firm chooses the stopping rate $s(p)$. Standard game-theoretic reasoning tells us that if $s(p) \in(0, \infty)$, then the firm must be indifferent between stopping and continuing. Therefore, fixing the worker's strategy, we can only focus on the firm's stopping strategies that are pure strategies. That is, $s(x) \in\{0, \infty\}$. In other words, the firm decides a stopping time $\tau$.

The potential difficulty is that the firm's stopping problem may not be well-behaved, as the worker's behavior-and thus the flow payoffs and volatility that the firm faces-can change discontinuously with the state. In spite of this problem, the next result allows one to verify that a given firm strategy is in fact a best response. The lemma is close to Theorem 10.4.1 from Øksendal (2003).

LEMMA 3: Let $a \in \mathcal{A}$. Suppose $\psi:[0,1] \rightarrow \mathbb{R}_{+}$and $\hat{p} \in[0,1]$ satisfy the following properties:

- $\psi$ is $C^{1}$ and piecewise $C^{2}$.

- $\left.\psi\right|_{[0, \hat{p}]}=0$.

- For a.e. $p \in[0,1]$,

$$
\psi(p)-\frac{1}{2}[a(p) p(1-p)]^{2} \psi^{\prime \prime}(p) \geq a(p) p-c
$$

with equality if $p>\hat{p}$.

Then $\psi=\pi\left(\cdot \mid s_{\hat{p}}, a\right)=\max _{s \in \mathcal{S}} \pi(\cdot \mid s, a)$. That is, $s_{\hat{p}}$ is an optimal strategy, yielding firm value function $\psi$.

\section{PROOF:}

Fix $p \in(\hat{p}, 1)$, and consider the belief process $\left\{P_{t}\right\}_{t}$ defined by $a \in \mathcal{A}$ and $P_{0}=p$.

Let $\tau^{*}:=\inf \left\{t: P_{t} \notin(\hat{p}, 1)\right\}$, and let $\tau$ be an arbitrary $\left\{P_{t}\right\}_{t}$-measurable (stochastic) stopping rule. 
By Itô's formula,

$$
\begin{aligned}
e^{-\tau \wedge n} \psi\left(P_{\tau \wedge n}\right)= & \psi(p)+\int_{0}^{\tau \wedge n} e^{-u}\left[-\psi\left(P_{u}\right)+\frac{1}{2}\left[a\left(P_{u}\right) P_{u}\left(1-P_{u}\right)\right]^{2} \psi^{\prime \prime}\left(P_{u}\right)\right] d u \\
& +\int_{0}^{\tau \wedge n} e^{-u} \psi^{\prime}\left(P_{u}\right)\left[a\left(P_{u}\right) P_{u}\left(1-P_{u}\right)\right] d B_{u} \\
\Rightarrow \psi(p)= & e^{-\tau \wedge n} \psi\left(P_{\tau \wedge n}\right)+\int_{0}^{\tau \wedge n} e^{-u}\left[\psi\left(P_{u}\right)-\frac{1}{2}\left[a\left(P_{u}\right) P_{u}\left(1-P_{u}\right)\right]^{2} \psi^{\prime \prime}\left(P_{u}\right)\right] d u \\
& -\int_{0}^{\tau \wedge n} e^{-u} \psi^{\prime}\left(P_{u}\right)\left[a\left(P_{u}\right) P_{u}\left(1-P_{u}\right)\right] d B_{u} .
\end{aligned}
$$

As the integrand of the stochastic integral is bounded, we can take expectations, yielding:

$$
\begin{aligned}
\psi(p) & =E e^{-\tau \wedge n} \psi\left(P_{\tau \wedge n}\right)+E \int_{0}^{\tau \wedge n} e^{-u}\left[\psi\left(P_{u}\right)-\frac{1}{2}\left[a\left(P_{u}\right) P_{u}\left(1-P_{u}\right)\right]^{2} \psi\left(P_{u}\right)\right] d u \\
& \geq E e^{-\tau \wedge n} \psi\left(P_{\tau_{n}}\right)+E \int_{0}^{\tau \wedge n} e^{-u}\left[a\left(P_{u}\right) P_{u}-c\right] d u .
\end{aligned}
$$

By dominated convergence,

$$
\psi(p) \geq E e^{-\tau} \psi\left(P_{\tau}\right)+E \int_{0}^{\tau} e^{-u}\left[a\left(P_{u}\right) P_{u}-c\right] d u
$$

As $\tau$ was arbitrary, we conclude that $\psi(p) \geq \sup _{s \in \mathcal{S}} \pi(p \mid a, s)$.

In the above argument, if $\tau=\tau^{*}$, then the inequalities hold with equality, so that

$$
\psi(p)=E e^{-\tau^{*}} \psi\left(P_{\tau^{*}}\right)+E \int_{0}^{\tau^{*}} e^{-u}\left[a\left(P_{u}\right) P_{u}-c\right] d u=0+\pi\left(p \mid a, s_{\hat{p}}\right) .
$$

Together, we have

$$
\psi(p) \leq \pi\left(p \mid a, s_{\hat{p}}\right) \leq \sup _{s \in \mathcal{S}} \pi(p \mid a, s) \leq \psi(p) .
$$

Recall that $p \in(\hat{p}, 1)$ was arbitrary, so that (by Lemma 1) $\pi\left(\cdot \mid a, s_{\hat{p}}\right)$ $=\sup _{s \in \mathcal{S}} \pi(\cdot \mid a, s)=\psi$ on $[\hat{p}, 1]$. On $[0, \hat{p}]$, we see $\pi\left(\cdot \mid a, s_{\hat{p}}\right)=0=\psi$.

Now, take any $p \in[0, \hat{p})$, and consider the belief process $\left\{P_{t}\right\}_{t}$ defined by $a \in \mathcal{A}$ and $P_{0}=p$. Let $\bar{\tau}:=\inf \left\{t: P_{t} \notin(0, \hat{p})\right\}$, and let $\tau$ be an arbitrary $\left\{P_{t}\right\}_{t}$-measurable (stochastic) stopping rule. Now,

$$
\begin{aligned}
E \int_{0}^{\tau} e^{-t}\left[a\left(P_{t}\right) P_{t}-c\right] d t & =E \int_{0}^{\tau \wedge \bar{\tau}} e^{-t}\left[a\left(P_{t}\right) P_{t}-c\right] d t+E \int_{\tau \wedge \bar{\tau}}^{\tau} e^{-t}\left[a\left(P_{t}\right) P_{t}-c\right] d t \\
& \leq E \int_{\tau \wedge \bar{\tau}}^{\tau} e^{-t}\left[a\left(P_{t}\right) P_{t}-c\right] d t=E \mathbf{1}_{\bar{\tau} \leq \tau} \int_{\bar{\tau}}^{\tau} e^{-t}\left[a\left(P_{t}\right) P_{t}-c\right] d t \\
& \leq E \mathbf{1}_{\bar{\tau} \leq \tau} e^{-\bar{\tau}} \sup _{s \in \mathcal{S}} \pi\left(P_{\tau} \mid a, s\right) d t=E \mathbf{1}_{\bar{\tau} \leq \tau} e^{-\bar{\tau}} \sup _{s \in \mathcal{S}} \pi(\hat{p} \mid a, s) d t \\
& =0 .
\end{aligned}
$$


As $\tau$ was arbitrary, we have $\sup _{s \in \mathcal{S}} \pi(\cdot \mid a, s) \leq 0$ on $[0, \hat{p})$ as well.

Since we know that $v$ is $C^{2}$ over any interval where $s$ is finite-valued and Lipschitz, the only difficulty is at the (isolated) discontinuities of $s$. In what follows, we show that smooth pasting obtains even at these problem points.

LEMMA 4: Let $p \in(0,1)$ be such that $s(p)$ is finite, $\bar{s}$ be an upper bound for $s$ in a neighborhood of $p$, and $\underline{\sigma} \in(0, \underline{a} p(1-p))$ a lower bound for volatility in the same neighborhood. Let $v=v(\cdot \mid a, s)$ for some fixed $a \in \mathcal{A}$, or let $v=\sup _{a \in \mathcal{A}} v(\cdot \mid a, s)$ :

(i) If $\psi:[0,1] \rightarrow \mathbb{R}$ is $C^{2}$ and convex, $\psi(p)=v(p)$, and $v(q)>\psi(q)$ for $q \neq p$ in some neighborhood of $p$, then $(1+\bar{s}) v(p) \geq 1+(1 / 2) \underline{\sigma}^{2} \psi^{\prime \prime}(p)$.

(ii) If $\psi:[0,1] \rightarrow \mathbb{R}$ is $C^{2}$ and concave, $\psi(p)=v(p)$, and $v(q)<\psi(q)$ for $q \neq p$ in some neighborhood of $p$, then $v(p) \leq 1+(1 / 2) \underline{\sigma}^{2} \psi^{\prime \prime}(p)$.

The proof of these results is based on the so-called viscosity approach to differential equations. While pre-existing results do not carry over directly, our proof closely mimics arguments from Pham (2009).

\section{PROOF:}

That $s(p)$ is finite and $\psi$ continuous imply $\psi(p)=v(p)>0$, so that $\psi>0$ near $p$.

Fix some $\delta>0$ small enough that $s \leq \bar{s}, \sigma \geq \underline{\sigma}$, and $0<\psi \neq v$ on $(p-\delta, p+\delta) \backslash\{p\}$. Fix some sequence $\left\{p_{m}\right\}_{m} \subseteq(p-\delta, p+\delta) \backslash\{p\}$ converging to $p$. Let $\eta_{m}:=v\left(p_{m}\right)-\psi\left(p_{m}\right)$, which converges to zero by continuity of $v$.

Let $\left\{P_{u}^{a, p_{m}}\right\}_{u}$ denote the belief process associated with control $a \in \mathcal{A}$ given $P_{0}^{a, p_{m}}=p_{m}$. Define the stopping time $\tau_{m}^{a}:=\inf \left\{u \geq 0:\left|P_{u}^{a, p_{m}}-p_{m}\right| \geq \delta / 2\right\}$ $\wedge \sqrt{\left|\eta_{m}\right|}$.

Given any $\zeta>0$, define a sequence of controls $\left\{a_{m}\right\}_{m} \subseteq \mathcal{A}$ as follows: if $v=v(\cdot \mid a, s)$, let $a_{m}:=a$; otherwise, if $v=\sup _{a \in \mathcal{A}} v(\cdot \mid a, s)$, let $a_{m}$ satisfy

$E\left[\int_{0}^{\tau_{m}^{a}} e^{-u} e^{-\int_{0}^{u} s\left(P_{\hat{u}}^{a_{m}, p_{m}}\right) d \hat{u}} d u+e^{-\tau_{m}^{a_{m}}} e^{-\int_{0}^{\tau_{m}^{a_{m}}} s\left(P_{\hat{u}}^{a_{m}, p_{m}}\right) d \hat{u}} v\left(P_{\tau_{m}^{a}}^{a_{m}, p_{m}}\right)\right] \geq v\left(p_{m}\right)-\zeta \sqrt{\left|\eta_{m}\right|}$,

which is achievable by some $a_{m}$ by the principle of optimality. As a shorthand, let $P^{m}:=P^{a_{m}, p_{m}}$ and $\tau_{m}:=\tau_{m}^{a_{m}}$.

For $r=1,1+\bar{s}$, Itô's formula delivers

$$
\begin{aligned}
e^{-r \tau_{m}} \psi\left(P_{\tau_{m}}^{m}\right)= & \psi\left(p_{m}\right)-r \int_{0}^{\tau_{m}} e^{-r u} \psi\left(P_{u}^{m}\right) d u \\
& +\int_{0}^{\tau_{m}} e^{-r u} \frac{1}{2}\left[a\left(P_{u}^{m}\right) P_{u}^{m}\left(1-P_{u}^{m}\right)\right]^{2} \psi^{\prime \prime}\left(P_{u}^{m}\right) d u
\end{aligned}
$$




$$
\begin{aligned}
& +\int_{0}^{\tau_{m}} e^{-r u} a\left(P_{u}^{m}\right)^{2} P_{u}^{m}\left(1-P_{u}^{m}\right)\left(\theta-P_{u}^{m}\right) \psi^{\prime}\left(P_{u}^{m}\right) d u \\
& +\int_{0}^{\tau_{m}} e^{-r u} a\left(P_{u}^{m}\right) P_{u}^{m}\left(1-P_{u}^{m}\right) \psi^{\prime}\left(P_{u}^{m}\right) d B_{u} .
\end{aligned}
$$

Taking expectations (note, $\psi$ is bounded), together with $\eta_{m} \psi^{\prime \prime} \geq 0$, gives us

$$
\begin{aligned}
\eta_{m} & \left\{E e^{-r \tau_{m}} \psi\left(P_{\tau_{m}}^{m}\right)-\psi\left(p_{m}\right)\right\} \\
& =\eta_{m}\left\{-r E \int_{0}^{\tau_{m}} e^{-r u} \psi\left(P_{u}^{m}\right) d u+E \int_{0}^{\tau_{m}} e^{-r u} \frac{1}{2}\left[a\left(P_{u}^{m}\right) P_{u}^{m}\left(1-P_{u}^{m}\right)\right]^{2} \psi^{\prime \prime}\left(P_{u}^{m}\right) d u\right\} \\
& \geq \eta_{m}\left\{-r E \int_{0}^{\tau_{m}} e^{-r u} \psi\left(P_{u}^{m}\right) d u+E \int_{0}^{\tau_{m}} e^{-r u} \frac{1}{2} \underline{\sigma}^{2} \psi^{\prime \prime}\left(P_{u}^{m}\right) d u\right\} \\
& =\eta_{m} E \int_{0}^{\tau_{m}} e^{-r u}\left[\frac{1}{2} \underline{\sigma}^{2} \psi^{\prime \prime}\left(P_{u}^{m}\right)-r \psi\left(P_{u}^{m}\right)\right] d u
\end{aligned}
$$

Now, we reason separately for the two cases.

First, suppose $\psi$ is convex and (around $p$ ) below $v$. Then,

$$
\begin{aligned}
\eta_{m} & =v\left(p_{m}\right)-\psi\left(p_{m}\right) \\
& \geq E\left[\int_{0}^{\tau_{m}} e^{-u} e^{-\int_{0}^{u} s\left(P_{\hat{u}}^{m}\right) d \hat{u}} d u+e^{-\tau_{m}} e^{-\int_{0}^{\tau_{m}} s\left(P_{\hat{u}}^{m}\right) d u} v\left(P_{\tau_{m}}^{m}\right)\right]-\psi\left(p_{m}\right) \\
& \geq E\left\{\int_{0}^{\tau_{m}} e^{-(1+\bar{s}) u} d u+\left[e^{-(1+\bar{s}) \tau_{m}} \psi\left(P_{\tau_{m}}^{m}\right)-\psi\left(p_{m}\right)\right]\right\} \\
& \geq E \int_{0}^{\tau_{m}} e^{-(1+\bar{s}) u}\left[1+\frac{1}{2} \underline{\sigma}^{2} \psi^{\prime \prime}\left(P_{u}^{m}\right)-(1+\bar{s}) \psi\left(P_{u}^{m}\right)\right] d u \\
\Rightarrow \sqrt{\eta_{m}} & \geq E \frac{\tau_{m}}{\sqrt{\eta_{m}}} \frac{1}{\tau_{m}} \int_{0}^{\tau_{m}} e^{-(1+\bar{s}) u}\left[1+\frac{1}{2} \underline{\sigma}^{2} \psi^{\prime \prime}\left(P_{u}^{m}\right)-(1+\bar{s}) \psi\left(P_{u}^{m}\right)\right] d u
\end{aligned}
$$

Taking limits as $m \rightarrow \infty$, (and applying the mean value theorem) tells us $0 \geq 1+(1 / 2) \underline{\sigma}^{2} \psi^{\prime \prime}(p)-(1+\bar{s}) v(p)$, as required.

Next, suppose $\psi$ is concave and (around $p$ ) above $v$.

By choice of $a_{m}$,

$$
\begin{aligned}
\eta_{m}-\zeta \sqrt{\left|\eta_{m}\right|} & =v\left(p_{m}\right)-\psi\left(p_{m}\right)-\zeta \sqrt{\left|\eta_{m}\right|} \\
& \leq E\left[\int_{0}^{\tau_{m}} e^{-u} e^{-\int_{0}^{u} s\left(P_{\hat{u}}^{m}\right) d \hat{u}} d u+e^{-\tau_{m}} e^{-\int_{0}^{\tau_{m}} s\left(P_{\hat{u}}^{m}\right) d \hat{u}} v\left(P_{\tau_{m}}^{m}\right)\right]-\psi\left(p_{m}\right) \\
& \leq E\left[\int_{0}^{\tau_{m}} e^{-u} d u+e^{-\tau_{m}} v\left(P_{\tau_{m}}^{m}\right)\right]-\psi\left(p_{m}\right) \\
& \leq E \int_{0}^{\tau_{m}} e^{-u}\left[1+\frac{1}{2} \underline{\sigma}^{2} \psi^{\prime \prime}\left(P_{u}^{m}\right)-\psi\left(P_{u}^{m}\right)\right] d u \\
\Rightarrow-\sqrt{\left|\eta_{m}\right|}-\zeta & \leq E \frac{\tau_{m}}{\sqrt{\eta_{m}}} \frac{1}{\tau_{m}} \int_{0}^{\tau_{m}} e^{-u}\left[1+\frac{1}{2} \underline{\sigma}^{2} \psi^{\prime \prime}\left(P_{u}^{m}\right)-\psi\left(P_{u}^{m}\right)\right] d u .
\end{aligned}
$$


Taking limits now yields $-\zeta \leq 1+(1 / 2) \underline{\sigma}^{2} \psi^{\prime \prime}(p)-v(p)$. Since this is true for arbitrary $\zeta>0$, we conclude that $v(p) \leq 1+(1 / 2) \underline{\sigma}^{2} \psi^{\prime \prime}(p)$, as required.

LEMMA 5: Let $p \in(0,1)$ be such that $s(p)$ is finite. Let $v=v(\cdot \mid a, s)$ for some fixed $a \in \mathcal{A}$, or let $v=\sup _{a \in \mathcal{A}} v(\cdot \mid a, s)$. Then $v$ is differentiable at $p$.

\section{PROOF:}

By Lemma $1, v$ is continuous at $p$. Then, by Lemma 2, both one-sided derivatives exist: call them $v_{-}^{\prime}(p)$ and $v_{+}^{\prime}(p)$ from the left and right, respectively. It remains to show that $v_{-}^{\prime}(p)=v_{+}^{\prime}(p)$.

Let $\bar{s} \in \mathbb{R}_{+}$be some local upper bound for $s$ around $p$, and let $\underline{\sigma} \in(0, p(1-p) \underline{a})$ a local lower bound for $q \mapsto q(1-q) a(q)$ around $p$. Take any $\lambda>0$.

If $\exists \kappa \in\left(v_{-}^{\prime}(p), v_{+}^{\prime}(p)\right)$, then letting $\psi(q):=v(p)+\kappa(q-p)+\lambda(q-p)^{2}$, Lemma 4(i) tells us that $v(p) \geq\left(1+\underline{\sigma}^{2} \lambda\right) /(1+\bar{s})$. If $\exists \kappa \in\left(v_{+}^{\prime}(p), v_{-}^{\prime}(p)\right)$, then letting $\psi(q):=v(p)+\kappa(q-p)-\lambda(q-p)^{2}$, Lemma 4(ii) tells us that $v(p) \leq 1-\underline{\sigma}^{2} \lambda$.

In either case, the making $\lambda$ arbitrarily large would violate $0 \leq v \leq 1$. So it must be that the one-sided derivatives match.

\section{B. Necessary Conditions for Equilibrium}

In this subsection, we collect some features that must be true of an arbitrary equilibrium. Together, these results will leave us with a unique candidate.

The following lemma is our first step towards characterizing the entire set of equilibria. We show that, as the worker wishes to be employed for as long as possible, he seeks to minimize the flow of information whenever his job is secure for the moment. Moreover, as the worker's behavior is consistent at such beliefs, the firm's behavior takes a similar form to that of a standard one-player bandit problem: the set of beliefs at which the worker is never fired will just be every belief above a certain cutoff.

LEMMA 6: In any unrefined equilibrium $(a, s)$, there is a cutoff $p_{H} \in(0,1)$ such that $\{p \in[0,1]: \pi(p)=0\}=\left[0, p_{H}\right]$, and $a_{\left(p_{H}, 1\right]}=\underline{a}$.

\section{PROOF:}

First we show the following claim: If $0 \leq p_{0}<p_{1} \leq 1$, and $\left(p_{0}, p_{1}\right)$ is a maximal interval $I$ such that $\pi(p)>0 \forall p \in I$, then $\left.a\right|_{\left(p_{0}, p_{1}\right)}=\underline{a}$, and $p_{1}=1$.

Firm best response implies $\left.s\right|_{\left(p_{0}, p_{1}\right)}$ is finite-valued and almost everywhere zero. Then, over any subinterval of $I$ where $v$ is $C^{2}$, we have

$$
v(p)=1+\frac{1}{2} p^{2}(1-p)^{2} \max _{a \in[\underline{a}, \bar{a}]}\left\{a^{2} v^{\prime \prime}(p)\right\} .
$$


For $\tilde{p} \approx 0$, the firm optimally stops irrespective of worker behavior. This implies $v<1$ globally, so that the above equation implies $v^{\prime \prime}(p)<0$. Therefore, choosing $a$ to maximize $a^{2} v^{\prime \prime}(p)$ is the same as minimizing $a^{2}$, which is uniquely attained by $a=\underline{a}$. So over any subinterval of $I$ where $v$ is $C^{2}$, we have $a=\underline{a}$ (almost everywhere). Then, Lemma 2 tells us that $I$ is the finite union of such subintervals, so that $a=\underline{a}$ (a.e.) on $I$.

Now, the firm's flow payoff is $f(p):=\underline{a} p-c$ for $p \in\left(p_{0}, p_{1}\right)$.

Fix $\tilde{p} \in\left(p_{0}, p_{1}\right)$. As $\pi$ is zero at any endpoint less than 1 (by maximality of the interval and Lemma 1), and belief 1 is a.s. never reached, it must be that

$$
\pi(\tilde{p})=\int_{p_{0}}^{p_{1}} f d \mu
$$

for some positive, absolutely continuous measure $\mu$ on $\left(z_{0}, z_{1}\right)$. As $\pi(\tilde{p})>0$, it must be that $f$ is positive on a set of positive measure. Since $f$ is increasing, it is positive in a neighborhood of $p_{1}$. Finally, applying Rüschendorf and Urusov (2008, Proposition 2.9), it cannot be optimal for the firm to stop at $p_{1}$ if $p_{1}<1$. That is, the firm would like to continue, despite a possibly negative flow payoff, to the right of $p_{1}$. This would contradict the maximality of $p_{1}$. Therefore, $p_{1}=1$, proving the claim.

Now, using the claim, we prove the lemma. First, notice that there is some $p \in(0,1)$ such that $\pi(p)>0$. Indeed, this property is guaranteed for $p>c / \underline{a}$, since such states are guaranteed strictly positive flow profit. Next, notice that any $p$ with $\pi(p)>0$ belongs to some open interval with the same property by Lemma 1 . The claim then tells us that $\left.\pi\right|_{[p, 1)}>0$ and $\left.a\right|_{[p, 1)}=\underline{a}$. Finally, let

$$
p_{H}:=\inf \{p \in[0,1]: \pi(p)>0\} .
$$

We now know that $\left.\pi\right|_{\left(p_{H}, 1\right)}>0,\left.a\right|_{\left(p_{H}, 1\right)}=\underline{a}$, and $\left.\pi\right|_{\left(0, p_{H}\right)}=0$. Lastly, by Lemma $1, \pi(0)=\pi\left(p_{H}\right)=0$ too.

LEMMA 7: In any unrefined equilibrium $(a, s)$, the firm's value function is $\pi(\cdot \mid a, s)=\sup _{\tilde{s} \in \mathcal{S}} \pi(\cdot \mid \underline{a}, \tilde{s})$.

\section{PROOF:}

Let $\pi:=\pi(\cdot \mid a, s)=\sup _{\tilde{s} \in \mathcal{S}} \pi(\cdot \mid a, \tilde{s})$ and $\pi_{*}:=\sup _{\tilde{s} \in \mathcal{S}} \pi(\cdot \mid \underline{a}, \tilde{s})$. First, we show that $\pi \geq \pi_{*} \cdot 35$

Fix any $p \in[0,1]$. Let $\left\{X_{t}, A_{t}\right\}_{t}$ be the stochastic process generated by $\left(P_{0}=p\right.$ and) worker strategy $a$; note that $A$ is $X$-measurable. Augmenting the probability space if needed, we may assume there is some Weiner process $\left\{\breve{B}_{t}\right\}_{t}$ which is independent of $(\theta, B, X, A)$.

\footnotetext{
${ }^{35}$ An alternative proof of this fact would adopt the viscosity approach to rule out existence of a convex kink in the firm's optimal value function.
} 
As the flow profit $\theta A_{t}-c$ is pathwise at least $\underline{a}$, we know that $\pi(p)$ is at least

$$
\check{\pi}:=\sup _{\tau}\left\{E \int_{0}^{\tau} e^{-t}(\underline{a} \theta-c) d t\right\} \quad \text { such that } \tau \text { is a stopping time adapted to } X \text {. }
$$

Indeed, they are optimal values from optimal stopping problems whose objectives are ranked for every stopping time.

Next, notice that $\check{\pi}$ is at least

$$
\check{\pi}_{*}:=\sup _{\tau}\left\{E \int_{0}^{\tau} e^{-t}(\underline{a} \theta-c) d t\right\} \quad \text { such that } \tau \text { is a stopping time adapted to } Y,
$$

where

$$
d Y:=\frac{a}{A}\left[d X+c d t+\frac{\sqrt{A^{2}-\underline{a}^{2}}}{\underline{a}} d \check{B}\right] .
$$

Indeed, by construction, any $Y$-adapted stopping time can be simulated by a (stochastic) $X$-adapted stopping time.

Finally, notice that $d Y_{t}=\theta \underline{a} d t+\left(\left(\underline{a} / A_{t}\right) d B_{t}+\left(\sqrt{A_{t}^{2}-\underline{a}^{2}} / A_{t}\right) d \check{B}\right)$, where the term in parentheses is itself the stochastic derivative of a Weiner process which is independent of $\theta$. In particular, the law of $\left\{Y_{t}\right\}_{t}$ conditional on $\theta$ is identical to the law that $\left\{X_{t}+t c\right\}_{t}$ would have under worker strategy $\underline{a} \in \mathcal{A}$. It follows that $\check{\pi}_{*}=\pi_{*}(p)$ (since the firm faces identical flow payoff and identical information across the two optimal stopping problems). Therefore, $\pi(p) \geq \check{\pi} \geq \check{\pi}_{*}=\pi_{*}(p)$.

Now, we prove the reverse inequality.

Let $p_{H} \in(0,1)$ be the cutoff guaranteed by Lemma 6. As $(a, s)$ is an unrefined equilibrium, $\pi$ is the firm's optimal value function given worker strategy $a$. Lemma 6 tells us that $\pi^{-1}(0)=\left[0, p_{H}\right]$, so that the simple cutoff strategy $s^{p_{H}}$ is also a firm best response to $a$. That is, $\pi\left(\cdot \mid a, s^{p_{H}}\right)=\pi$. But $\pi\left(\cdot \mid a, s^{p_{H}}\right)=\pi\left(\cdot \mid \underline{a}, s^{p_{H}}\right)$, as they feature exactly the same behavior on path. Therefore, $\pi=\pi\left(\cdot \mid a, s^{p_{H}}\right)=\pi\left(\cdot \mid \underline{a}, s^{p_{H}}\right) \leq \pi_{*}$.

Proposition 1 follows immediately from Lemma 7.

NOTATION 1: Let $\delta:=(1 / 2)(\sqrt{1+(1 / 8 \underline{a})}-1), \bar{p}:=c \delta /(\underline{a}(1+\delta)-c)$, and $\underline{p}:=\min \{\bar{p}, c / \bar{a}\}$.

LEMMA 8: Take any unrefined equilibrium $(a, s)$.

The simple cutoff strategy $s^{\bar{p}} \in \mathcal{S}$ is a best response to $\underline{a} \in \mathcal{A}$, and the value function $\pi=\pi(\cdot \mid \underline{a}, s)$ satisfies

$$
\pi(p)=\left[\underline{a} p-c+(c-\underline{a} \bar{p})\left(\frac{p}{\bar{p}}\right)^{-\delta}\left(\frac{1-p}{1-\bar{p}}\right)^{1+\delta}\right] \mathbf{1}_{p \geq \bar{p}} .
$$

PROOF:

Let $\psi$ be the proposed function on the right-hand side. 
On $(\bar{p}, 1], \psi$ is $C^{2}$ and satisfies $\psi(p)=\underline{a} p-c+(1 / 2)[\underline{a} p(1-p)]^{2} \psi^{\prime \prime}(p)$. On $[0, \bar{p}), \psi$ is zero and flow payoffs are less than or equal to 0 . Lastly, $\psi$ is differentiable at $\bar{p}$. Lemma 3 then tells us that $\psi=\sup _{\tilde{s} \in \mathcal{S}} \pi(\cdot \mid \underline{a}, \tilde{s})$, and $s^{\bar{p}}$ is optimal for the firm against $\underline{a}$. That $\pi=\psi$ then follows from Lemma 7 .

LEMMA 9: In any unrefined equilibrium $(a, s)$, on any open interval $I \subseteq[0, \bar{p}]$ where the worker has a strictly positive value, the worker uses $a(p)=c / p$.

\section{PROOF:}

Consider any $p \in I$ at which $a$ is continuous. Note that $s(p)<\infty$ because $v(p)>0$. So consider an $\epsilon>0$ and $\bar{s} \in \mathbb{R}_{+}$such that $(p-\epsilon, p+\epsilon) \subseteq I$ and $\left.s\right|_{(p-\epsilon, p+\epsilon)} \leq \bar{s}$, and consider the belief process $\left\{P_{t}\right\}_{t}$ associated with worker control $a$ and $P_{0}=p$. Let $\tau_{\epsilon}:=\inf \left\{t:\left|P_{t}-p\right| \geq \epsilon\right\}$. We can decompose $\pi(p)$ as follows:

$$
\begin{aligned}
0 & =\pi(p) \\
& =E\left[\int_{0}^{\tau_{\epsilon}} e^{-t} e^{-\int_{0}^{t} s\left(P_{u}\right) d u}\left[a\left(P_{t}\right) P_{t}-c\right] d t+e^{-\tau_{\epsilon}} e^{-\int_{0}^{\tau_{\epsilon}} s\left(P_{u}\right) d u} \pi\left(P_{\tau_{\epsilon}}\right)\right] \\
& =E \int_{0}^{\tau_{\epsilon}} e^{-t} e^{-\int_{0}^{t} s\left(P_{u}\right) d u}\left[a\left(P_{t}\right) P_{t}-c\right] d t \\
\Rightarrow 0 & =E \frac{1}{\tau_{\epsilon}} \int_{0}^{\tau_{\epsilon}} e^{-t} e^{-\int_{0}^{t} s\left(P_{u}\right) d u}\left[a\left(P_{t}\right) P_{t}-c\right] d t, \quad \text { where } e^{-\int_{0}^{t} s\left(P_{u}\right) d u} \in\left[e^{-\bar{s} t}, 1\right] .
\end{aligned}
$$

Taking limits as $\epsilon \rightarrow 0$ and appealing to the mean value theorem, $a(p) p-c=0$. This condition holds at every $p \in I$ at which $a$ is continuous, and so it holds almost everywhere, as required.

LEMMA 10: In any unrefined equilibrium $(a, s)$, on any open interval I $\subseteq[0, \bar{p}]$ where the worker has a strictly positive value, $v(p)$ is affine.

\section{PROOF:}

Since $\left.v\right|_{I}>0$, we know $\left.s\right|_{I}$ is finite-valued.

Consider an open subinterval $J \subseteq I \backslash\{\underline{a} / c\}$ over which $s$ is Lipschitz. By Lemma 2, for $p \in J$,

$$
a(p) v^{\prime \prime}(p)=\frac{[1+s(p)] v(p)-1}{\frac{1}{2} p(1-p)}=\sup _{\hat{a} \in[\underline{a}, \bar{a}]}\left[\hat{a} v^{\prime \prime}(p)\right] .
$$

As $a(p) \neq \underline{a}, \bar{a}$, it must be that $v^{\prime \prime}(p)=0$.

The above tells us that, if $s$ is piecewise Lipschitz over a subinterval $J$ of $I$, then $v$ is piecewise affine over said subinterval. Moreover, by Lemma $5,\left.v\right|_{J}$ is then affine.

Therefore, $v$ is affine in a neighborhood of any $p \in I$. As a result, it is affine over $I$. 
LEMMA 11: In any unrefined equilibrium $(a, s)$, there is a cutoff $p_{L}$ such that the worker's zero value set is $\left[0, p_{L}\right]$.

\section{PROOF:}

Consider any $\hat{p} \in(0,1)$ with $v(p)>0$. Let us show that $\left.v\right|_{[\hat{p}, 1]}>0$. In the proof below, we will use Lemma 1 many times.

First, note that $\hat{p}$ belongs to some maximal open interval $\left(p_{0}, p_{1}\right)$ with the property that $\left.v\right|_{\left(p_{0}, p_{1}\right)}>0$. We know that the firm best response requires stopping for sufficiently low beliefs, so that $p_{0}>0$. By the intermediate value theorem, $v\left(p_{0}\right)=0$. By Lemma 10, $v$ takes the form $v(p)=\gamma\left(p-p_{0}\right)$ for $p \in\left(p_{0}, p_{1}\right)$, where $\gamma \in \mathbb{R}$ is some constant. As $v(\hat{p})>0$, it must be that $\gamma>0$, implying $v\left(p_{1}\right)>0$. But then (continuity of $v$ and) maximality of $p_{1}$ implies that $p_{1}=1$. So $\left.v\right|_{[\hat{p}, 1]}>0$.

Letting $p_{L}:=\inf \{p \in[0,1]: v(p)>0\}$ concludes the proof.

LEMMA 12: Take any unrefined equilibrium $(a, s)$, and let $p_{L}$ be as delivered by Lemma 11. Then $(a, s)$ is an equilbrium if and only if $\left.a\right|_{\left(0, p_{L}\right)}=\bar{a}$.

\section{PROOF:}

For the proof of this lemma, it is convenient to work with log-likelihood ratios rather than beliefs. For $z \in \mathbb{R}$, let $p(z):=1 /\left(1+e^{-z}\right)$, so that $z=\log (p(z) /(1-p(z)))$. Let $\hat{v}(z):=v(p(z) \mid a, s)$ for any $z \in \mathbb{R}$.

Let $z_{L}:=\log \left(p_{L} /\left(1-p_{L}\right)\right)$, and take any $z_{0}<z_{L}$. We want to show that instantaneous sequential rationality implies $a\left(p\left(z_{0}\right)\right)=\bar{a}$. To this end, fix arbitrary $\hat{a} \in[\underline{a}, \bar{a})$.

For any $a_{0} \in[\underline{a}, \bar{a}]$, let $\left\{Z_{t}^{a_{0}}\right\}_{t}$ be the $X$-measurable stochastic process for log-likelihood ratios associated with initial condition $Z_{0}^{a_{0}}=z_{0}$ and constant control $a_{0} \in \mathcal{A}$. That is, $Z^{a_{0}}$ satisfies the $\operatorname{SDE}$ (see equation 1 )

$$
d Z_{t}^{a_{0}}=a_{0}\left[a_{0}\left(\theta-\frac{1}{2}\right) d t+d B_{t}\right]
$$

Crucially, the " $d B_{t}$ " term is the same for every different $a_{0}$, so that these stochastic processes are correlated.

To begin with, we prove the following claim: There are constants $\gamma, \hat{\Delta}>0$ such that, for every $\Delta \in(0, \hat{\Delta}]$ and $a_{0} \in[\underline{a}, \hat{a}]$, we have

$$
Z_{\Delta}^{\bar{a}} \geq z_{L}+\gamma \quad \text { if } Z_{\Delta}^{a_{0}} \geq z_{L}
$$

To see this, observe that

$$
\begin{aligned}
d Z_{t}^{\bar{a}}-d Z_{t}^{a_{0}} & =\bar{a}\left[\bar{a}\left(\theta-\frac{1}{2}\right) d t+d B_{t}\right]-a_{0}\left[a_{0}\left(\theta-\frac{1}{2}\right) d t+d B_{t}\right] \\
& =\left(\bar{a}^{2}-a_{0}^{2}\right)\left(\theta-\frac{1}{2}\right) d t+\left(\bar{a}-a_{0}\right) d B_{t} \\
& =\bar{a}\left(\bar{a}-a_{0}\right)\left(\theta-\frac{1}{2}\right) d t+\frac{\bar{a}-a_{0}}{a_{0}} d Z_{t}^{a_{0}}
\end{aligned}
$$




$$
\begin{gathered}
\geq-\frac{\bar{a}}{2}(\bar{a}-\underline{a}) d t+\frac{\bar{a}-a_{0}}{a_{0}} d Z_{t}^{a_{0}} \\
\Rightarrow Z_{\Delta}^{\bar{a}} \geq Z_{\Delta}^{a_{0}}-\frac{\bar{a}}{2}(\bar{a}-\underline{a}) \Delta+\frac{\bar{a}-a_{0}}{a_{0}}\left(Z_{\Delta}^{a_{0}}-z_{0}\right) .
\end{gathered}
$$

In particular, if $Z_{\Delta}^{a_{0}} \geq z_{L}>z_{0}$, then

$$
\begin{aligned}
Z_{\Delta}^{\bar{a}} & \geq Z_{\Delta}^{a_{0}}-\frac{\bar{a}}{2}(\bar{a}-\underline{a}) \Delta+\frac{\bar{a}-a_{0}}{a_{0}}\left(Z_{\Delta}^{a_{0}}-z_{0}\right) \\
& \geq z_{L}-\frac{\bar{a}}{2}(\bar{a}-\underline{a}) \Delta+\frac{\bar{a}-\hat{a}}{\hat{a}}\left(z_{L}-z_{0}\right) .
\end{aligned}
$$

Taking any $\gamma \in\left(0,((\bar{a}-\hat{a}) / \hat{a})\left(z_{L}-z_{0}\right)\right)$ with small enough $\hat{\Delta}>0$ will witness the claim.

We now use the claim to prove the lemma.

Notice next that there is a constant $M \geq 0$ such that $\hat{v}(z) \leq M\left(z-z_{L}\right)_{+}$for all $z \in \mathbb{R}$. Indeed, $v(\cdot \mid a, s)$ is affine on $\left[p_{L}, \bar{p}\right]$ by Lemma 10 , concave on $[\bar{p}, 1]$ by the proof of Lemma 6 , and differentiable at $\bar{p}$ if $\bar{p}>p_{L}$ by Lemma 5 ; therefore it is concave on $[\bar{p}, 1]$. Next, the right-hand derivative $m$ of said function is finite-by Lemma 10 if $\bar{p}>p_{L}$, by the proof of Lemma 6 and direct computation if $\bar{p}=p_{L}$. Moreover, these same two lemmas tell us that the function is increasing on $\left[p_{L}, 1\right]$, so that (being constant on $\left[0, p_{L}\right]$ ) it is Lipschitz of constant $m$. Then, as a composition of Lipschitz functions, $\hat{v}$ is itself Lipschitz of some constant $M$. The bound then follows directly.

Now, for any $a_{0} \in[\underline{a}, \hat{a}]$ and $\Delta \in(0, \hat{\Delta}]$ :

$$
\begin{aligned}
\frac{E \hat{v}\left(Z_{\Delta}^{a_{0}}\right)}{E \hat{v}\left(Z_{\Delta}^{\bar{a}}\right)} & \leq \frac{E \hat{v}\left(Z_{\Delta}^{a_{0}}\right)}{E\left[\hat{v}\left(Z_{\Delta}^{\bar{a}}\right) \mathbf{1}_{\left.\left\{Z_{\Delta}^{\bar{a}} \geq z_{L}+\gamma\right\}\right]}\right.} \\
& \left.=\frac{\operatorname{Pr}\left[Z_{\Delta}^{a_{0}} \geq z_{L}\right] E\left[\hat{v}\left(Z_{\Delta}^{a_{0}}\right) \mid Z_{\Delta}^{a_{0}} \geq z_{L}\right]}{\operatorname{Pr}\left[Z_{\Delta}^{\bar{a}} \geq z_{L}+\gamma\right] E\left[\hat{v}\left(Z_{\Delta}^{\bar{a}}\right) \mid Z_{\Delta}^{\bar{a}} \geq z_{L}+\gamma\right]} \quad \text { (since } \hat{v} \text { is zero on }\left[0, z_{L}\right]\right) \\
& \leq \frac{E\left[\hat{v}\left(Z_{\Delta}^{a_{0}}\right) \mid Z_{\Delta}^{a_{0}} \geq z_{L}\right]}{E\left[\hat{v}\left(Z_{\Delta}^{\bar{a}}\right) \mid Z_{\Delta}^{\bar{a}} \geq z_{L}+\gamma\right]} \quad(\text { by the claim }) \\
& \leq \frac{E\left[\hat{v}\left(Z_{\Delta}^{a_{0}}\right) \mid Z_{\Delta}^{a_{0}} \geq z_{L}\right]}{\hat{v}\left(z_{L}+\gamma\right)} \quad(\text { by monotonicity of } v) \\
& \leq \frac{M}{\hat{v}\left(z_{L}+\gamma\right)} E\left[Z_{\Delta}^{a_{0}}-z_{L} \mid Z_{\Delta}^{a_{0}} \geq z_{L}\right] .
\end{aligned}
$$


Conditional on $\theta \in\{0,1\}$, the random variable $Z_{\Delta}^{a_{0}}-z_{L}$ is normally distributed with mean $-\left(z_{L}-z_{0}\right)+a_{0}^{2}(\theta-(1 / 2)) \Delta$ and standard deviation $a_{0} \sqrt{\Delta}$. Therefore, applying the known formula for the expectation of truncated normal:

$$
\begin{aligned}
E_{\theta} & {\left[Z_{\Delta}^{a_{0}}-z_{L} \mid Z_{\Delta}^{a_{0}}-z_{L} \geq 0\right] } \\
& =E_{\theta}\left[Z_{\Delta}^{a_{0}}-z_{L}\right]+\operatorname{std}_{\theta}\left[Z_{\Delta}^{a_{0}}-z_{L}\right] \cdot \frac{\phi}{1-\Phi}\left(\frac{0-E_{\theta}\left[Z_{\Delta}^{a_{0}}-z_{L}\right]}{\operatorname{std}_{\theta}\left[Z_{\Delta}^{a_{0}}-z_{L}\right]}\right) \\
& =-\left(z_{L}-z_{0}\right)+a_{0}^{2}\left(\theta-\frac{1}{2}\right) \Delta+a_{0} \sqrt{\Delta} \cdot \frac{\phi}{1-\Phi}\left(\frac{z_{L}-z_{0}-a_{0}^{2}\left(\theta-\frac{1}{2}\right) \Delta}{a_{0} \sqrt{\Delta}}\right) \\
& =\left[\left(z_{L}-z_{0}\right)-a_{0}^{2}\left(\theta-\frac{1}{2}\right) \Delta\right]\left[-1+\epsilon_{\Delta}^{a_{0}, \theta} \frac{\phi}{1-\Phi}\left(\frac{1}{\epsilon_{\Delta}^{a_{0}, \theta}}\right)\right],
\end{aligned}
$$

where $\epsilon_{\Delta}^{a_{0}, \theta}:=a_{0} \sqrt{\Delta} /\left(z_{L}-z_{0}-a_{0}^{2}(\theta-(1 / 2)) \Delta\right)$. Notice that $\epsilon_{\Delta}^{a_{0}, \theta}>0$ for sufficiently small $\Delta$, and that $\lim _{\Delta \rightarrow 0} \sup _{a_{0} \in[\underline{a}, \bar{a}]} \epsilon_{\Delta}^{a_{0}, \theta}=0$. Now, observe that

$$
\begin{aligned}
\lim _{\epsilon \rightarrow 0} \epsilon \frac{\phi}{1-\Phi}\left(\frac{1}{\epsilon}\right)=\lim _{\epsilon \rightarrow 0} \frac{\epsilon e^{-\frac{1}{2 \epsilon^{2}}}}{\int_{1 / \epsilon}^{\infty} e^{-\frac{y^{2}}{2}} d y} & =\lim _{\epsilon \rightarrow 0} \frac{e^{-\frac{1}{2 \epsilon^{2}}}\left[1+\epsilon\left(-\frac{1}{2}\right)(-2) \epsilon^{-3}\right]}{-e^{-\frac{1}{2 \epsilon^{2}}}\left(-\epsilon^{-2}\right)} \text { (by L'Hopital) } \\
& =\lim _{\epsilon \rightarrow 0} \frac{1+\epsilon^{-2}}{\epsilon^{-2}}=\lim _{\epsilon \rightarrow 0}\left(1+\epsilon^{2}\right)=1 \Rightarrow \\
\lim _{\Delta \rightarrow 0} \sup _{a_{0} \in[\underline{a}, \bar{a}]} E_{\theta}\left[Z_{\Delta}^{a_{0}}-z_{L} \mid Z_{\Delta}^{a_{0}} \geq z_{L}\right] & =\left(z_{L}-z_{0}\right)\left[-1+\lim _{\Delta \rightarrow 0} \sup _{a_{0} \in[\underline{a}, \bar{a}]} \epsilon_{\Delta}^{a_{0}, \theta} \frac{\phi}{1-\Phi}\left(\frac{1}{\epsilon_{\Delta}^{a_{0}, \theta}}\right)\right] \\
& =0 .
\end{aligned}
$$

Finally, this tells us that

$$
\begin{aligned}
& \lim _{\Delta \rightarrow 0} \sup _{a_{0} \in[\underline{a}, \hat{a}]} \frac{E \hat{v}\left(Z_{\Delta}^{a_{0}}\right)}{E \hat{v}\left(Z_{\Delta}^{\bar{a}}\right)} \leq \frac{M}{\hat{v}\left(z_{L}+\gamma\right)} \lim _{\Delta \rightarrow 0} \sup _{a_{0} \in[\underline{a}, \bar{a}]} E\left[Z_{\Delta}^{a_{0}}-z_{L} \mid Z_{\Delta}^{a_{0}} \geq z_{L}\right] \\
& \leq \frac{M}{\hat{v}\left(z_{L}+\gamma\right)} \lim _{\Delta \rightarrow 0} \max _{\theta \in\{0,1\}_{a_{0} \in[a, \bar{a}]}} \sup _{\theta} E_{\theta}\left[Z_{\Delta}^{a_{0}}-z_{L} \mid Z_{\Delta}^{a_{0}} \geq z_{L}\right]=0 .
\end{aligned}
$$

Therefore, for sufficiently small $\Delta>0$, we have $\sup _{a_{0} \in[a, \hat{a}} E \hat{v}\left(Z_{\Delta}^{a_{0}}\right)<E \hat{v}\left(Z_{\Delta}^{\bar{a}}\right)$. That is, $a_{\Delta}^{*}(p \mid a, s)=\operatorname{argmax}_{a_{0} \in[a, \bar{a}]} E \hat{v}\left(Z_{\Delta}^{a_{0}}\right) \subseteq(\hat{a}, \bar{a}]$. But then $\hat{a} \in[\underline{a}, \bar{a})$ was arbitrary.

We are now equipped to prove both directions of the lemma, given $p \in\left(0, p_{L}\right)$. First, given any $a_{0} \in[\underline{a}, \bar{a})$, the set $a_{\Delta}^{*}(p \mid a, s)$ is contained in $\left[\left(\bar{a}+a_{0}\right) / 2, \bar{a}\right]$ for sufficiently small $\Delta$, so that $a(p) \neq a_{0}$ if $(a, s)$ is to be an equilibrium. Second, it is straightforward that the maximum defining $a_{\Delta}^{*}(p \mid a, s)$ is attained by some $a_{\Delta} \in[\underline{a}, \bar{a}]$. But then $a_{\Delta}>\hat{a}$ for sufficiently small $\Delta$ for any $\hat{a} \in[\underline{a}, \bar{a})$. Therefore $a_{\Delta} \rightarrow \bar{a}$ as $\Delta \rightarrow 0$, proving instantaneous sequential rationality of $\bar{a}$. 
LEMMA 13: Take any equilibrium $(a, s)$, and let $p_{L}$ be as delivered by Lemma 11. Then $p_{L}=\underline{p}$.

\section{PROOF:}

First, suppose that $p_{L}<\bar{p}$. For all $p \in\left(p_{L}, \bar{a}\right)$, we know from Lemma 9 that $\bar{a} \geq a(p)=c / p$, so that $p \geq c / \bar{a}$. Considering $p$ very close to $p_{L}$ then tells us that $p_{L} \geq c / \bar{a}$.

So either $p_{L} \geq \bar{p}$ or $p_{L} \geq c / \bar{a}$. That is, $p_{L} \geq p$.

Next, by Lemma 8, the firm has strictly positive value above $\bar{p}$, and so does not stop there. Therefore, the worker has strictly positive value above $\bar{p}$ too, so that $p_{L} \leq \bar{p}$. All that remains is to show that $p_{L} \leq c / \bar{a}$.

By firm best response, there cannot be an open subinterval of $\left[0, p_{L}\right]$ on which the firm's flow profit $p \mapsto a(p) p-c$ is strictly positive. For $p$ in such an interval, the associated flow profit is simply $\bar{a} p-c$. Focusing on such intervals contained in a small neighborhood of $p_{L}$, it must be that $\bar{a} p_{L}-c \leq 0$, as desired.

\section{Sufficient Conditions for Equilibrium}

In this subsection, we lay the groundwork to show that our candidate equilibrium is in fact an equilibrium.

LEMMA 14: There is a unique continuous function $v:[0,1] \rightarrow \mathbb{R}$ such that:

(i) v is $C^{2}$ on $[0,1] \backslash\{\underline{p}, \bar{p}\}$ and differentiable on $(\underline{p}, 1]$.

(ii) $\left.v\right|_{[0, \underline{p}]}=0$ and $v(1)=1$.

(iii) $v$ is affine on $[\underline{p}, \bar{p}]$ and satisfies $v(p)=1+(1 / 2)[\underline{a} p(1-p)]^{2} v^{\prime \prime}(p)$ for $p \in(\bar{p}, 1)$.

Moreover, this function is concave and strictly increasing over $(\underline{a}, 1)$, and so $(0,1)$-valued there.

PROOF:

Given any function $v$ of the desired form, there are constants $\beta, K$ such that ${ }^{36}$

$$
v(p)= \begin{cases}0 & \text { if } p \in[0, \underline{p}] \\ \beta(p-\underline{p}) & \text { if } p \in(\underline{p}, \bar{p}] . \\ 1-K(1-p){ }^{1+\delta} p^{-\delta} & \text { if } p \in[\bar{p}, 1]\end{cases}
$$

\footnotetext{
${ }^{36}$ Notice that the second order linear DE over $(\bar{p}, 1)$ has solutions $p \mapsto 1-K^{\prime} p^{1+\delta}(1-p)^{-\delta}-K(1-p)^{1+\delta} p^{-\delta}$ for constants $K, K^{\prime}$. But then continuity and $v(1)=1$ imply $K^{\prime}=0$. Similarly, the form for $v$ over $(\underline{p}, \bar{p}]$ comes from affinity together with continuity at $p$.
} 
If $p=\bar{p}$ (so that $\beta$ is irrelevant), we need to see that there is a unique constant $K$ such that the value matching equation $1-K(1-\bar{p})^{1+\delta} \bar{p}^{-\delta}=0$ holds. This is clearly so, with $K=(1-\bar{p})^{-(1+\delta)} \bar{p}^{\delta}$.

If $\underline{p}<\bar{p}$, we need to see that there is a unique pair of constants $(\beta, K)$ such that the value matching and smooth pasting equations

$$
\begin{aligned}
\beta(\bar{p}-\underline{p}) & =1-K(1-\bar{p})^{1+\delta} \bar{p}^{-\delta}, \\
\beta & =K(1-\bar{p})^{\delta} \bar{p}^{-(1+\delta)}[p+\delta]
\end{aligned}
$$

hold. The solution set is the intersection of a positively sloped line and a negatively sloped line in $(\beta, K)$ space, delivering a unique solution.

Finally, direct computation shows that $\beta=(\bar{p}+\delta) /(\bar{p}(1-\underline{p})+\delta(\bar{p}-\underline{p}))$ $>0$, and $K=(1-\bar{p})^{-\delta} \bar{p}^{1+\delta}[p+\delta]^{-1} \beta>0$, so that $\left.v\right|_{[\underline{a}, 1]}$ is increasing and concave.

NOTATION 2: Define $a^{*} \in \mathcal{A}$ and $s^{*} \in \mathcal{S}$ as follows:

$$
\begin{aligned}
& a^{*}= \begin{cases}\underline{a} \text { if } p \in[\bar{p}, 1] \\
\underline{c} \text { if } p \in(\underline{p}, \bar{p}), \\
\bar{a} \text { if } p \in[0, \underline{p}]\end{cases} \\
& s^{*}= \begin{cases}0 & \text { if } p \in[\bar{p}, 1] \\
\frac{1-v(p)}{v(p)} & \text { if } p \in(\underline{p}, \bar{p}), \\
\infty & \text { if } p \in[0, \underline{p}]\end{cases}
\end{aligned}
$$

where $v$ is the function specified in Lemma 14.

LEMMA 15: Let $A:[0,1] \rightrightarrows[\underline{a}, \bar{a}]$ be given by either $A(p)=[\underline{a}, \bar{a}] \forall p$ or $A(p)=\left\{a^{*}(p)\right\} \forall p$. Then there is a unique continuous function $f:[0,1] \rightarrow[0,1]$ such that:

(i) fis $C^{2}$ on $[0,1] \backslash\{\underline{p}, \bar{p}\}$ and differentiable on $(\underline{p}, 1]$.

(ii) $\left.f\right|_{[0, \underline{p}]}=0$ and $f(1)=1$.

(iii) f satisfies the HJB

$$
\left[1+s^{*}(p)\right] f(p)=1+\frac{1}{2} \sup _{a \in A(p)}\left\{[a p(1-p)]^{2} f^{\prime \prime}(p)\right\}
$$




$$
\text { for } p \in(\underline{p}, \bar{p}) \cup(\bar{p}, 1) \text {. }
$$

\section{PROOF:}

Let $v$ be the function specified in Lemma 14. By that lemma, and by definition of $s^{*}$, the function $v$ satisfies the conditions of the present lemma. Let $f$ be any such function, and let $h:=f-v$. We must show that $h=0$.

First, notice that $h$ is continuous on $[0,1]$, differentiable on $(\underline{p}, 1)$, zero on $[0, \underline{p}] \cup\{1\}$, and $C^{2}$ on $[0,1] \backslash\{\underline{p}, \bar{p}\}$.

$$
\begin{aligned}
\text { For } p \in(\bar{p}, 1) & \\
1 & \geq f(p)=1+\frac{1}{2} \sup _{a \in A(p)}\left\{[a p(1-p)]^{2} f^{\prime \prime}(p)\right\} \\
\Rightarrow 0 & \geq \sup _{a \in A(p)}\left\{[a p(1-p)]^{2} f^{\prime \prime}(p)\right\} \Rightarrow 0 \geq f^{\prime \prime}(p) \\
\Rightarrow f(p) & =1+\frac{1}{2}[\underline{a} p(1-p)]^{2} f^{\prime \prime}(p) \\
\Rightarrow h(p) & =\frac{1}{2}[\underline{a} p(1-p)]^{2} h^{\prime \prime}(p) .
\end{aligned}
$$

For $p \in(\underline{p}, \bar{p})$,

$$
\begin{aligned}
{\left[1+s^{*}(p)\right] f(p) } & =1+\frac{1}{2} \sup _{a \in A(p)}\left\{[a p(1-p)]^{2}\left[f^{\prime \prime}(p)-0\right]\right\} \\
& =\left[1+s^{*}(p)\right] h(p)+\frac{1}{2} \sup _{a \in A(p)}\left\{[\operatorname{ap}(1-p)]^{2} h^{\prime \prime}(p)\right\} \\
\Rightarrow\left[1+s^{*}(p)\right] h(p) & =\frac{1}{2} \sup _{a \in A(p)}\left\{[\operatorname{ap}(1-p)]^{2} h^{\prime \prime}(p)\right\} .
\end{aligned}
$$

Take any subinterval $I$ of $(p, 1)$ on which $h$ is strictly positive [negative]. By the above equations, it is strictly convex [concave] over each of $I \cap(\underline{p}, \bar{p})$ and $I \cap(\bar{p}, 1)$. But then differentiability of $h$ on $I$ tells us that it is strictly convex [concave] over $I$.

Assume, for contradiction, that $h$ is nonzero. Lemma 1 then lets us say more.

There is then some maximal interval $\left(p_{0}, p_{1}\right) \subseteq(0,1)$ over which it is nonzero. As $\left.h\right|_{[0, p] \cup\{1\}}=0$, it must be that $h\left(p_{0}\right)=h\left(p_{1}\right)=0$, and $\left.h\right|_{\left(p_{0}, p_{1}\right)}$ is either strictly positive or strictly negative. By the above argument, $\left.h\right|_{\left(p_{0}, p_{1}\right)}$ is either positive and convex or negative and concave. This contradicts $h\left(p_{0}\right)=h\left(p_{1}\right)=0$.

\section{Proof of Theorem 1}

\section{PROOF:}

Consider any equilibrium. Lemmas 6 and 8 tell us that, above $\bar{p}$, the worker chooses the low action and the firm never fires. Lemmas 11, 12, and 13 tell us that, below $p$, the worker chooses the high action and the firm immediately fires. Lemmas $9, \overline{10}$, and 13 tell us that, over $(\underline{p}, \bar{p})$, the worker chooses an action to give 
zero flow payoff to the firm, and the worker's value is affine in beliefs. Lemma 14 then tells us that the firm's firing behavior agrees with $s^{*}$ over this interval. Summing up, if there is any equilibrium, then it is $\left(a^{*}, s^{*}\right)$.

We now verify that our unique candidate is in fact an equilibrium. Lemma 3 tells us that $s^{p}$ is a firm best response to $a^{*}$ for every $p \in[\underline{p}, \bar{p}]$. It follows that the mixture $s^{*}$ is as well.

Lastly, consider the two worker value functions $v\left(\cdot \mid a^{*}, s^{*}\right)$ and $\sup _{a \in \mathcal{A}} v\left(\cdot \mid a, s^{*}\right)$. Both are functions $[0,1] \rightarrow[0,1]$ which take value 0 over $[0, \underline{p}]$ and 1 at 1 . By Lemma 1 , both are continuous. By Lemma 2 , both are $C^{2}$ on $(\underline{p}, \bar{p}) \cup(\bar{p}, 1)$. By Lemma 5, both are differentiable at $\bar{p}$. Therefore, by Lemma 15, they coincide: $a^{*}$ is a worker best response to $s^{*}$ on path. Finally, Lemma 12 tells us that $a^{*}$ is instantaneously sequentially rational against $s^{*}$.

\section{E. Proof of Corollary 1}

\section{PROOF:}

Let $\hat{v}:=v(\bar{p})$. The belief function $v \mapsto p(v)$ is simply the inverse of the strictly increasing function $\left.v^{*}\right|_{(p, 1)}$. On $p \in(\underline{p}, \bar{p}), a^{*}(p) p-c=0$ by definition of $a^{*}$. Therefore, $F$ is zero over $[0, \hat{v})$.

For $p \in[\bar{p}, 1], v^{*}(p)=1-K(1-p)^{1+\delta} p^{-\delta}$ for $K>0$, so that $v^{*}$ is concave and increasing there. Therefore, its inverse is convex and increasing. Finally, $p \mapsto \underline{a} p-c$ is affine, increasing, negative at $p=\underline{a}$, and positive at $p=1$. Therefore, $F$ takes the desired form over $(\hat{v}, 1)$.

\section{REFERENCES}

Aghion, Philippe, and Matthew O. Jackson. 2016. "Inducing Leaders to Take Risky Decisions: Dismissal, Tenure, and Term Limits." American Economic Journal: Microeconomics 8 (3): 1-38.

-Anderson, Axel, and Luís M.B. Cabral. 2007. "Go for Broke or Play it Safe? Dynamic Competition with Choice of Variance." RAND Journal of Economics 38 (3): 593-609.

-Board, Simon, and Moritz Meyer-ter-Vehn. 2013. "Reputation for Quality." Econometrica 81 (6): 2381-2462.

Bohren, J. Aislinn. 2019. "Persistence in a Dynamic Moral Hazard Game.” https://static1.squarespace. com/static/5a1b26c580bd5e0fc6dbd478/t/5d8269b3066c1e0546a0b2e9/1568827839164/Bohren_ PersistenceDynamicMoralHazardGame_Sept2019.pdf.

Bolton, Patrick, and Christopher Harris. 1999. "Strategic Experimentation." Econometrica 67 (2): 349-74.

Brockner, Joel, Tom R. Tyler, and Rochelle Cooper-Schneider. 1992. "The Influence of Prior Commitment to an Institution on Reactions to Perceived Unfairness: The Higher They Are, the Harder They Fall." Administrative Science Quarterly 37 (2): 241-61.

Chaves, Isaías N. 2019. "Privacy in Bargaining: The Case of Endogenous Entry." https://isachavesv. github.io/papers/bargainingPaper.pdf.

-Cisternas, Gonzalo. 2018a. "Two-Sided Learning and the Ratchet Principle." Review of Economic Studies 85 (1): 307-51.

-Cisternas, Gonzalo. 2018b. "Career Concerns and the Nature of Skills." American Economic Journal: Microeconomics 10 (2): 152-89.

-Crandall, Michael G., Hitoshi Ishii, and Pierre-Louis Lions. 1992. "User's Guide to Viscosity Solutions of Second Order Partial Differential Equations." Bulletin of the American Mathematical Society 27 (1): 1-67.

Daley, Brendan, and Brett Green. 2012. "Waiting for News in the Market for Lemons." Econometrica 80 (4): 1433-1504. 
-Daley, Brendan, and Brett Green. 2020. "Bargaining and News." American Economic Review $102(2): 428-74$.

Demarzo, Peter M., and Yuliy Sannikov. 2017. "Learning, Termination, and Payout Policy in Dynamic Incentive Contracts." Review of Economic Studies 84 (1): 182-236.

Deming, W. Edwards. 1982. Out of the Crisis. Cambridge: MIT Center for Advanced Educational Services.

-Dilmé, Francesc. 2019. "Dynamic Quality Signaling with Moral Hazard." Games and Economic Behavior 113 (January): 116-36.

Dixit, Avinash. 1993. The Art of Smooth Pasting: Fundamentals of Pure and Applied Economics. New York: Routledge.

-Engellandt, Axel, and Regina T. Riphahn. 2005. "Temporary Contracts and Employee Effort." Labour Economics 12 (3): 281-99.

Garfagnini, Umberto. 2011. "Delegated Experimentation.” Unpublished.

Gilboa, Simona, Arie Shirom, Yitzhak Fried, and Cary Cooper. 2008. "A Meta-analysis of Work Demand Stressors and Job Performance: Examining Main and Moderating Effects." Personnel Psychology 61 (2): 227-71.

-Holmström, Bengt. 1999. "Managerial Incentive Problems: A Dynamic Perspective." Review of Economic Studies 66 (1): 169-82.

-Holmstrom, Bengt, and Paul Milgrom. 1991. "Multitask Principal-Agent Analyses: Incentive Contracts, Asset Ownership, and Job Design." Journal of Law, Economics, and Organization 7: $24-52$.

Ichino, Andrea, and Regina T. Riphahn. 2005. "The Effect of Employment Protection on Worker Effort: Absenteeism During and After Probation." Journal of the European Economic Association 3 (1): $120-43$.

-Jacob, Brian A. 2013. "The Effect of Employment Protection on Teacher Effort." Journal of Labor Economics 31 (4): 727-61.

-Kolb, Aaron M. 2015. “Optimal Entry Timing.” Journal of Economic Theory 157: 973-1000.

-Kolb, Aaron M. 2019. "Strategic Real Options." Journal of Economic Theory 183 (September): 344-83.

Kostadinov, Rumen, and Aditya Kuvalekar. Forthcoming. "Learning in Relational Contracts." American Economic Journal: Microeconomics.

Krylov, N.V. 2009. Controlled Diffusion Processes. Berlin: Springer.

-Lazear, Edward P. 1990. "Job Security Provisions and Employment." Quarterly Journal of Economics 105 (3): 699-726.

-Levin, Jonathan. 2003. "Relational Incentive Contracts." American Economic Review 93 (3): 835-57.

Nakao, Shintaro. 1972. "On the Pathwise Uniqueness of Solutions of One-Dimensional Stochastic Differential Equations." Osaka Journal of Mathematics 9 (3): 513-18.

- -ksendal, Bernt, ed. 2003. "Stochastic Differential Equations." In Stochastic Differential Equations: An Introduction with Applications, 65-84. Berlin: Springer.

Orlov, Dmitry, Andrzej Skrzypacz, and Pavel Zryumov. Forthcoming. "Persuading the Principal to Wait." Journal of Political Economy.

- Ortner, Juan. 2019. "A Continuous-Time Model of Bilateral Bargaining." Games and Economic Behavior 113: 720-33.

Pham, Huyên. 2009. Continuous-Time Stochastic Control and Optimization with Financial Applications: Stochastic Modelling and Applied Probability, Vol. 61. Berlin: Springer.

-Prat, Julien, and Boyan Jovanovic. 2014. "Dynamic Contracts when the Agent's Quality Is Unknown." Theoretical Economics 9 (3): 865-914.

-Probst, Tahira M., Susan M. Stewart, Melissa L. Gruys, and Bradley W. Tierney. 2007. "Productivity, Counterproductivity and Creativity: The Ups and Downs of Job Insecurity." Journal of Occupational and Organizational Psychology 80 (3): 479-97.

- Reisel, William D., Tahira M. Probst, Swee-Lim Chia, Cesar M. Maloles, and Cornelius J. König. 2010. "The Effects of Job Insecurity on Job Satisfaction, Organizational Citizenship Behavior, Deviant Behavior, and Negative Emotions of Employees." International Studies of Management and Organization 40 (1): 74-91.

Repenning, Nelson P. 2000. "Drive Out Fear (Unless You Can Drive It In): The Role of Agency and Job Security in Process Improvement." Management Science 46 (11): 1385-96.

- Roskies, Ethel, and Christiane Louis-Guerin. 1990. "Job Insecurity in Managers: Antecedents and Consequences." Journal of Organizational Behavior 11 (5): 345-59.

- Rüschendorf, Ludger, and Mikhail A. Urusov. 2008. "On a Class of Optimal Stopping Problems for Diffusions with Discontinuous Coefficients.” Annals of Applied Probability 18 (3): 847-78. 
-Sannikov, Yuliy. 2007. "Games with Imperfectly Observable Actions in Continuous Time." Econometrica 75 (5): 1285-1329.

Selenko, Eva, Anne Mäkikangas, Saija Mauno, and Ulla Kinnunen. 2013. "How Does Job Insecurity Relate to Self-Reported Job Performance? Analysing Curvilinear Associations in a Longitudinal Sample." Journal of Occupational and Organizational Psychology 86 (4): 522-42.

Shapiro, Carl, and Joseph E. Stiglitz. 1984. "Equilibrium Unemployment as a Worker Discipline Device." American Economic Review 74 (3): 433-44.

-Simon, Leo K., and Maxwell B. Stinchcombe. 1989. "Extensive Form Games in Continuous Time: Pure Strategies." Econometrica 57 (5): 1171-1214.

-Staufenbiel, Thomas, and Cornelius J. König. 2010. "A Model for the Effects of Job Insecurity on Performance, Turnover Intention, and Absenteeism." Journal of Occupational and Organizational Psychology 83 (1): 101-17.

Strulovici, Bruno, and Martin Szydlowski. 2015. "On the Smoothness of Value Functions and the Existence of Optimal Strategies in Diffusion Models." Journal of Economic Theory 159 (Part B): 1016-55.

-Sverke, Magnus, and Johnny Hellgren. 2001. "Exit, Voice and Loyalty Reactions to Job Insecurity in Sweden: Do Unionized and Non-unionized Employees Differ?" British Journal of Industrial Relations 39 (2): 167-82.

- Varas, Felipe. 2018. "Managerial Short-Termism, Turnover Policy, and the Dynamics of Incentives." Review of Financial Studies 31 (9): 3409-51.

Varas, Felipe, Iván Marinovic, and Andrzej Skrzypacz. 2019. "Random Inspections and Periodic Reviews: Optimal Dynamic Monitoring.” https://web.stanford.edu/ skrz/certification_design.pdf. 